ARTICLE

https://doi.org/10.1038/s41467-019-12522-w

\title{
Rare mutations in the complement regulatory gene CSMD1 are associated with male and female infertility
}

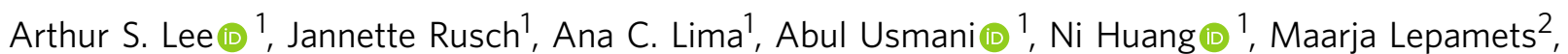
Katinka A. Vigh-Conrad ${ }^{3}$, Ronald E. Worthington ${ }^{4}$, Reedik Mägi ${ }^{2}$, Xiaobo Wu ${ }^{5}$, Kenneth I. Aston ${ }^{6}$, John P. Atkinson (10 ${ }^{5}$, Douglas T. Carrell ${ }^{6}$, Rex A. Hess (i) ${ }^{7}$, Moira K. O'Bryan (i) ${ }^{8}$ \& Donald F. Conrad (1) ${ }^{1,3,9 *}$

Infertility in men and women is a complex genetic trait with shared biological bases between the sexes. Here, we perform a series of rare variant analyses across 73,185 women and men to identify genes that contribute to primary gonadal dysfunction. We report CSMD1, a complement regulatory protein on chromosome $8 \mathrm{p} 23$, as a strong candidate locus in both sexes. We show that CSMD1 is enriched at the germ-cell/somatic-cell interface in both male and female gonads. Csmd7-knockout males show increased rates of infertility with significantly increased complement C3 protein deposition in the testes, accompanied by severe histological degeneration. Knockout females show significant reduction in ovarian quality and breeding success, as well as mammary branching impairment. Double knockout of Csmd1 and C3 causes non-additive reduction in breeding success, suggesting that CSMD1 and the complement pathway play an important role in the normal postnatal development of the gonads in both sexes.

\footnotetext{
${ }^{1}$ Department of Genetics, Washington University School of Medicine, St. Louis, MO 63110, USA. ${ }^{2}$ Estonian Genome Center, University of Tartu, 51010 Tartu, Estonia. ${ }^{3}$ Oregon National Primate Center, Oregon Health and Science University, Beaverton, OR 97006, USA. ${ }^{4}$ Department of Pharmaceutical Sciences, Southern Illinois University, Edwardsville, IL 62025, USA. ${ }^{5}$ Division of Rheumatology, Department of Medicine, Washington University School of Medicine, St. Louis, MO 63110, USA. ${ }^{6}$ Department of Surgery, University of Utah School of Medicine, Salt Lake City, UT 84132, USA. ${ }^{7}$ College of Veterinary Medicine, University of Illinois, Urbana-Champaign, IL 61802, USA. ${ }^{8}$ The School of Biological Sciences, Monash University, Clayton, Victoria 3800, Australia.

9 Department of Molecular and Medical Genetics, Oregon Health and Sciences University, Portland, OR 97239, USA. *email: conradon@ohsu.edu
} 
$\mathrm{T}$ he human genetic study of infertility has historically classified male infertility and female infertility as distinct diseases, leading to the assembly of many separate cohorts for the study of sex-specific reproductive processes ${ }^{1-4}$. However, numerous molecular and physiological mechanisms of fertility regulation are shared between male and female mammals ${ }^{5}$. For instance, programmed germ cell degeneration is a pervasive part of gonadal biology in both sexes ${ }^{6,7}$. In human males, roughly $80 \%$ of the meiotic descendants of spermatogonial stem cells undergo apoptosis prior to ever becoming mature spermatozoa ${ }^{8}$. Likewise, human females are born with $\sim 500,000$ oocytes and ovulate roughly 400 times after menarche. However, only roughly 1000 oocytes survive the sojourn to menopause, representing colossal germ cell loss not attributable to ovulation ${ }^{9}$. This ratio of surviving:apoptotic germ cells differs between species, but is narrowly maintained within species ${ }^{8,10}$. The principles that govern the loss versus survival of given germ cells are poorly understood.

Defects in germ cell development intrinsic to the gonad are defined as primary gonadal dysfunction. Primary gonadal dysfunction - which manifests as early idiopathic menopause in women and oligo-/azoo-spermia in men - is a subset of the infertility phenotype that is tractable for human genetic analysis and has a prevalence of at least $1 \%$ in adult males and females ${ }^{11,12}$. We previously identified a reproducible association between rare copy number variant $(\mathrm{CNV})$ burden and oligospermia and azoo-spermia in men ${ }^{13,14}$. One of several candidate loci from this study included the complement regulatory CUB and Sushi multiple domains 1 (CSMD1) gene.

The primary protein sequence of CSMD1 shares homology with complement-interacting proteins ${ }^{15}$. Complement acts as an inflammatory/phagocytic signal in the innate immune system ${ }^{16}$, and previous work has shown that classical complement components $\mathrm{C} 1 \mathrm{q}$ and $\mathrm{C} 3$ are also responsible for microglia-mediated phagocytosis of excess neuronal cells in a normal post-natal developmental process known as synaptic pruning ${ }^{17}$. Common variants in CSMD1 (refs. ${ }^{18,19}$ ) and complement $C 4$ (ref. ${ }^{20}$ ) have also been associated with schizophrenia in independent, wellpowered human association studies. Furthermore, some of the most significantly associated variants previously associated with azoospermia include the greater MHC locus, which encompasses complement $C 2, C 4$, and factor $\mathrm{B}^{21,22}$. CSMD1 is also known to inhibit the classical complement pathway in vitro ${ }^{15,23}$.

In this study, we incorporate array and exome sequencing data from a large cohort of early idiopathic menopause from the Women's Health Initiative ${ }^{24}$ to search for novel, shared factors required for normal gonadal function in both sexes, identifying an association with the gene CSMD1 in male and female gonadal dysfunction, and replicate our findings with samples from the UK Biobank $^{25}$. We characterize the expression patterns of CSMD1 in female and male gonads and measure morphological and reproductive traits in Csmd1-knockout mice, thereby adding a level of functional support to our genetic findings. Finally, to consolidate the putative roles of complement with Csmd1-mediated pathology, we investigate the in situ abundance of macrophages and complement component C3 in Csmd1-wildtype versus Csmd1null tissues. Our findings highlight the value of human genetic analysis as an entry point into surprising and previously unappreciated pathways in disease processes - in this case, a novel connection between of complement-related processes and gonadal function.

\section{Results}

CSMD1 is associated with gonadal failure in both sexes. Due to the strong purifying selection against infertility mutations, we hypothesized that male and female gonadal dysfunction are driven largely by rare mutation events. To test this hypothesis, we obtained SNP array and phenotype data from 12,002 women (515 cases of early idiopathic menopause vs. 11,487 normal menopause controls) and 2,072 men (321 cases of oligo-spermia or azoospermia vs. 1,751 normospermic controls) with documented reproductive health history. Due to reduced linkage disequilibrium between common SNPs and rare variants, we instead leveraged the SNP $\log$ R ratios and B-allele frequencies to perform $\mathrm{ab}$ initio discovery of CNVs that occupy the entire allele frequency spectrum (Supplementary Data 1). We performed rigorous call and sample QC with emphasis on high confidence calls based on established methods (see Methods section ${ }^{13,14,26}$, Supplementary Fig. 1). We then applied filters to enrich for rare, deleterious CNVs. We used these CNVs to perform a gene-based, case-control rare variant association separately in males and females. This approach allows for interrogation across the genome with a substantially lower multiple hypothesis burden than is required for traditional common-variant GWAS.

Our rare variant association study identified a significant association between early idiopathic menopause and deletions overlapping the CSMD1 gene located on chromosome 8p23.2 $\left(\mathrm{OR}=16\right.$; PLINK nominal $p$-value $=4.0 \times 10^{-4}$; PLINK genomewide $p$-value $=0.015$; Fig. 1, Supplementary Tables 1, 2, Supplementary Data 2). This association signal replicated in GEMINI $^{13}$, our smaller cohort of male oligo-/azoo-spermia cases $\left(\mathrm{OR}=3.3\right.$; PLINK nominal $p$-value $\left.=6.5 \times 10^{-3}\right)$. This CNV association is driven by an enrichment of rare deletions in cases, all of which are clustered at the $5^{\prime}$ end of the gene within introns 1-3 (5'-deletions; Fig. 1a).

To further replicate the association between deletions in CSMD1 and risk for gonadal dysfunction, we assembled an independent female early idiopathic menopause case-control cohort using the UK Biobank (see Methods section). After CNV $\mathrm{QC}$ and rigorous case/control selection, we obtained a cohort of 63,064 women with both reliable phenotype data and CNV calls (1,873 cases versus 59,947 controls). We again observed a significant association between early menopause and rare $5^{\prime}$ deletions of CSMD1 $(\mathrm{OR}=3.03$, logistic regression $p$-value $<5 \times$ $10^{-4}$, Fig. 1a, b). To summarize the overall risk conferred by rare CSMD1 5'-deletions of all sizes, we performed a meta-analysis across all three cohorts $(\mathrm{OR}=3.695 \%$ CI [2.14-6.01]; metaanalysis logistic regression $p$-value $=1.2 \times 10^{-6}$; Fig. 1c; Supplementary Table 1).

We further tested our association using genotypes ascertained by an orthogonal genotyping modality, by analyzing rare (MAF < 0.01) CSMD1 single nucleotide variants (SNVs) from exome sequencing performed on a subset of the WHI cohort $(n=1526)$. We performed a single-locus quantitative trait analysis and found a statistically significant association between rare, deleterious CSMD1 SNVs and age at menopause (SKAT single-locus $p$-value $<5 \times 10^{-3}$; Fig. 1, see Methods section). The CSMD1 protein product consists almost entirely of alternating/repeating CUB (complement $\mathrm{C} 1 \mathrm{r} / \mathrm{C} 1 \mathrm{~s}$, Uegf, Bmp1) and Sushi/CCP (complement control protein) domains (Fig. 2a). Thus, in order to assess the relative contribution of in CUB versus Sushi domain SNVs to age at menopause, we fit linear models to partition the association signal among these two domains. The CSMD1 SNV association was driven almost exclusively by mutations in the CUB $\left(\beta_{\mathrm{CUB}}=\right.$ $-0.86)$, but not Sushi $\left(\beta_{\mathrm{SUSHI}}=0.046\right)$ domains (Wilcoxon ranksum test $p$-value $=0.043$; Fig. 1d, see Methods section). Our regression model estimates that each CUB domain SNV accelerates the onset of menopause by $\sim 10$ months. Finally, a well-powered common variant GWAS in a female cohort of 182,416 individuals identified 3 common SNPs within CSMD1 to be significantly and independently associated with age at menarche and subsequently replicated in $>300,000$ 
a

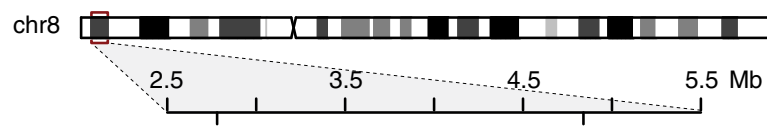

CSMD1

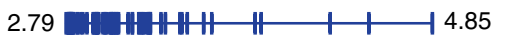

Variants found

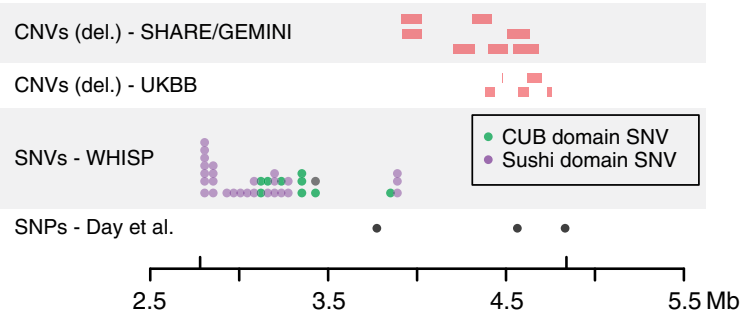

b

CNVs (deletions)

\begin{tabular}{lcccccccc}
\hline \multirow{2}{*}{ Trait } & Cohort & cases & controls & cases & $\begin{array}{c}N \text { deletions } \\
\text { controls }\end{array}$ & $\begin{array}{c}\text { Adjusted } \\
\text { OR }\end{array}$ & $\begin{array}{c}\text { Nominal } \\
p \text {-value }\end{array}$ \\
\hline Early menopause & SHARE & 515 & 11,487 & 3 & 3 & 16 & $4.00 \mathrm{E}-04$ \\
Azoospermia & GEMINI & 321 & 1751 & 5 & 7 & 3.3 & $6.50 \mathrm{E}-03$ \\
Early menopause & UKBB & 1873 & 59,947 & 11 & 116 & 3 & $5.00 \mathrm{E}-04$ \\
\hline
\end{tabular}

EXOME (SNVs)

\begin{tabular}{lcccc}
\hline Trait & Cohort & $N$ samples & $N$ SNVs & SKAT $p$-value \\
\hline Age at menopause & WHISP & 1526 & 30 & $5.00 \mathrm{E}-03$ \\
\hline
\end{tabular}

GWAS (SNPs)

\begin{tabular}{lccccc}
\hline \multirow{2}{*}{ Trait } & Cohort & $N$ samples & rsID & y/allele & $p$-value \\
\hline Age at menarche & Day et al. & 326,391 & rs2688326 & -0.0364 & $4.34 \mathrm{E}-18$ \\
Age at menarche & Day et al. & 326,392 & rs2724961 & -0.0459 & $3.76 \mathrm{E}-33$ \\
Age at menarche & Day et al. & 326,392 & rs4875424 & -0.0332 & $1.99 \mathrm{E}-16$
\end{tabular}

C

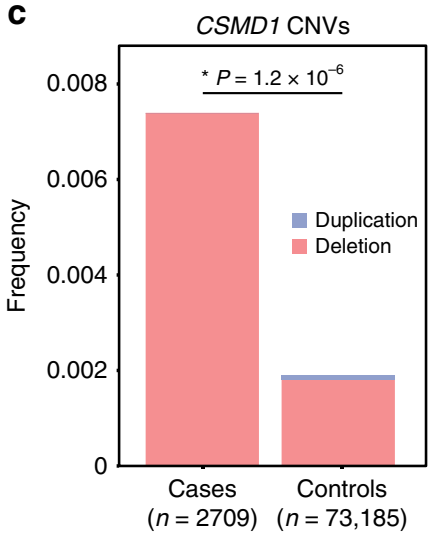

d

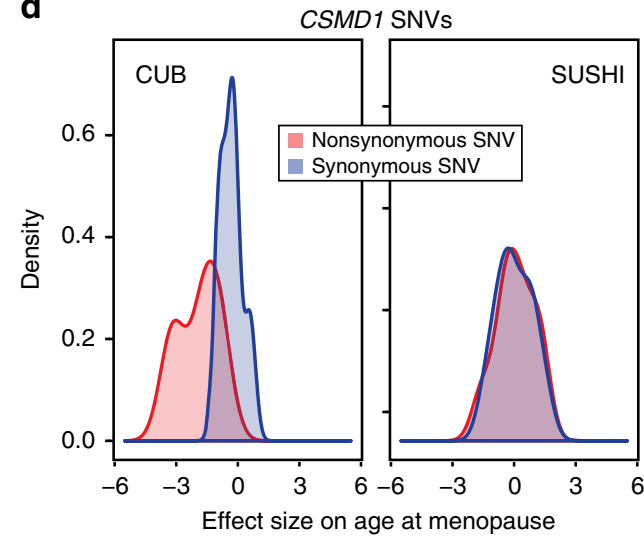

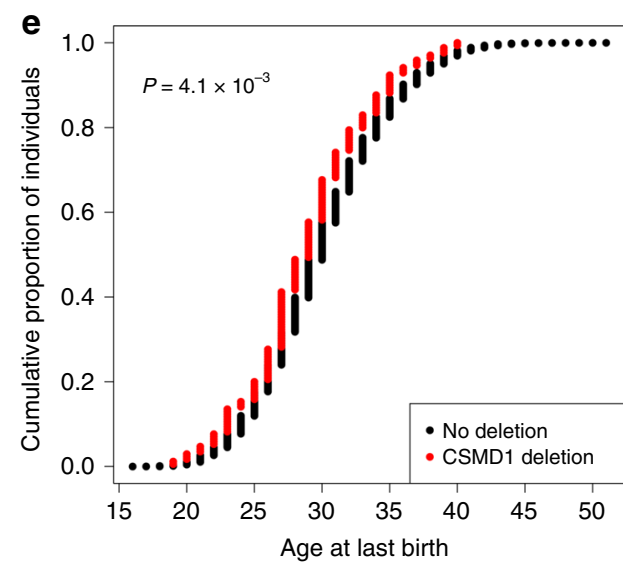

Fig. 1 Genetic variants in CSMD1 are associated with primary gonadal dysfunction. a The landscape of genetic variation across CSMD1 among all cohorts. SHARE/GEMINI: rare deletions overlapping CSMD1 among 14,074 females and males from the SHARE and GEMINI cohorts; UKBB: rare intron 1-3 deletions observed in 63,064 females from the UK Biobank early idiopathic menopause cohort - for clarity of visualization only the deletions observed in cases are shown; WHISP: 37 rare SNVs overlapping CSMD1 among 1,526 exome-sequenced females; and Day et al.: three statistically independent lead SNPs from a large-scale GWAS of age at menarche in females are depicted as points along the bottom of the figure (left to right): rs2688326, rs2724961, and rs $4875424^{28}$. All coordinates are in hg19. b Summary of the human genetic evidence that CSMD1 variants are associated with gonadal function in men and women described in this study. c Stacked barplot depicting frequency of rare CNVs overlapping introns 1-3 of CSMD1 among 2,709 cases of male or female gonadal dysfunction versus 73,185 controls. Rare deletions over CSMD1 segregate significantly with cases (odds ratio = 3.6; meta-analysis logistic regression $p$-value $=1.2 \times 10^{-6}$ ). $\mathbf{d}$ Density plots depicting effect sizes $(\beta)$ of rare CSMD1 SNVs on age at menopause for synonymous (blue) and nonsynonymous (red) SNVs, stratified by protein domain (CUB vs. Sushi). SNVs in the CUB domains are significantly associated with an earlier onset of menopause when compared to SNVs in the Sushi domains ( $\beta_{\mathrm{CUB}}=-0.86,95 \% \mathrm{Cl}[-1.56,-0.151] ; \beta_{\mathrm{SUSHI}}=0.046,95 \% \mathrm{Cl}[-0.255,0.377]$; Wilcoxon rank-sum test $p$-value $=0.043$ ). Synonymous mutations in both CUB and Sushi domains are centered about 0 , consistent with a well-calibrated estimate of effect size. e The cumulative distribution of age at last birth in UK Biobank subjects, plotted separately by CSMD1 deletion status. There is a 1-year reduction in the mean age at last birth for women with deletions in introns 1-3 of CSMD1 compared to women without deletions (linear regression $p$-value $=4.1 \times 10^{-3}$ ). Source data are provided as a Source Data file

individuals 27,28 (Fig. 1). Subsequent work in the same cohort showed age of menarche and menopause to be positively correlated. Furthermore, the common CSMD1 SNPs associated with age of menarche also correctly predicted age of menopause in the expected direction $\left(\beta_{\mathrm{rs} 2688325}=0.014 ; \beta_{\mathrm{rs} 7828501}=0.021\right.$; $\left.\beta_{\text {rs7463166 }}=0.031\right)^{29}$.

The phenotype of idiopathic early menopause indicates problems in ovarian function. Predictable consequences of such ovarian dysfunction are irregular or absent menses and reduced fecundity. To further clarify the role of CSMD1 deletions in female reproductive traits, we tested for association with 4 additional reproductive phenotypes available from the UKBB (see Methods section). Of these traits, we identified a significant association with age at last live birth $(n=76,686$, linear regression $p$-value $=4.1 \times 10^{-3}$, Fig. $\left.1 \mathrm{e}\right)$. Individuals with CSMD1 deletions show a reduced reproductive lifespan across all quartiles of the cumulative distribution, corresponding to an average reduction of 1 year. To compare relative effect sizes, our fitted model predicts that obesity status reduces reproductive lifespan by 0.6 years, and smoking status reduces reproductive lifespan by 1 year (see Methods section). The maximum age at last birth among our controls is 51 years, versus 40 years for CSMD1 deletion carriers. However, we note that $99.6 \%$ of control individuals have completed childbearing by age 36 , indicating that traditional measures of fertility (e.g. fecundity) would not necessarily capture ovarian dysfunction related to CSMD1 deletions.

In summary, we detected associations between rare variants in CSMD1 and gonadal dysfunction (i) across multiple classes of 


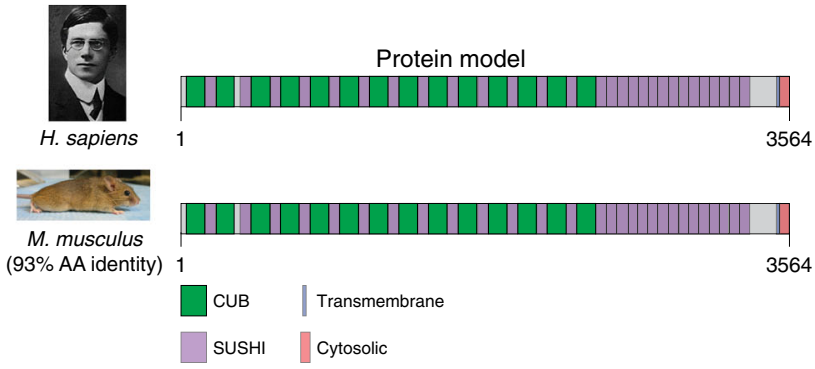

C
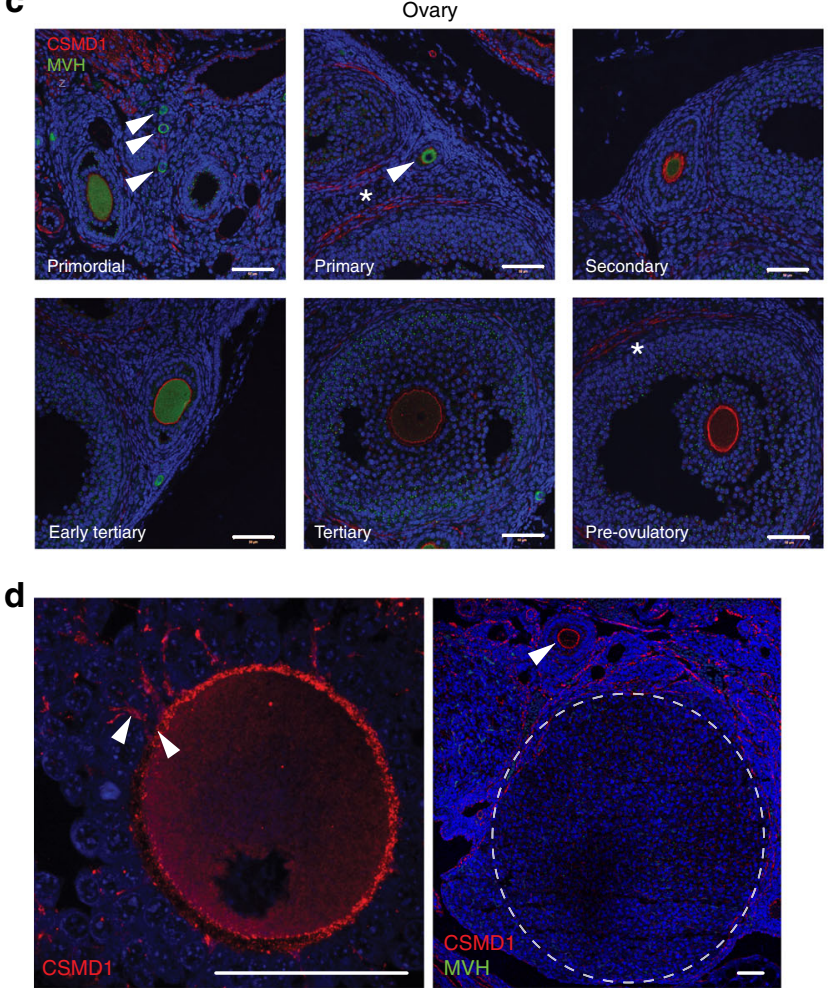

b
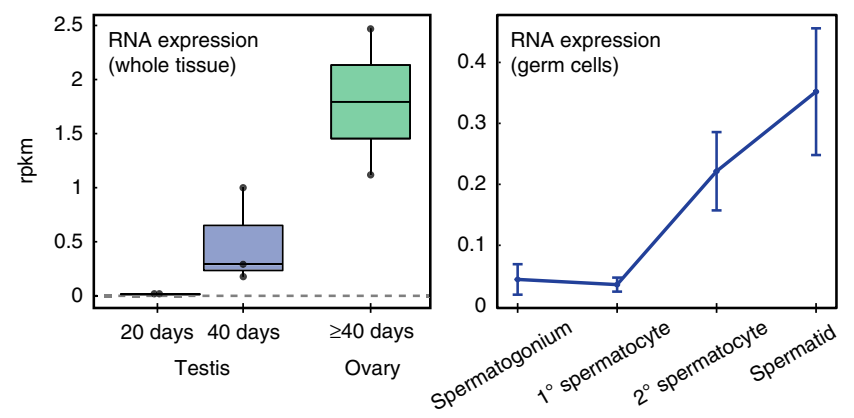

e

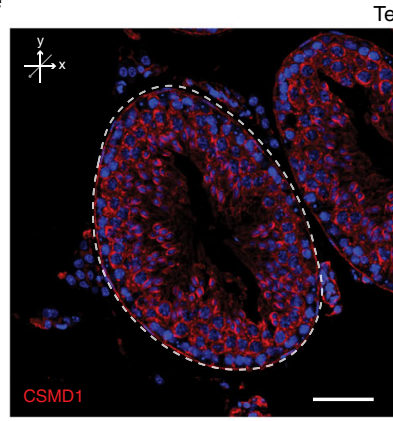

Testis
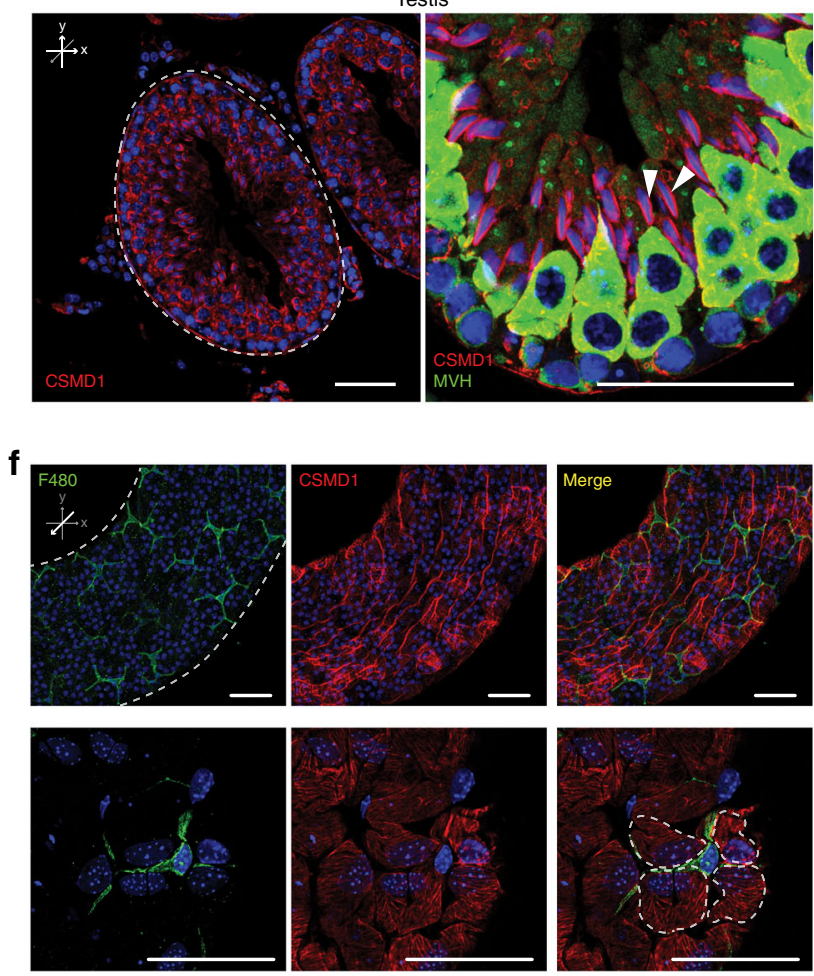

Fig. 2 Csmd1 is expressed in the male and female gonads. a Protein model of CSMD1 in human and mouse. CUB and Sushi domains, as well as the transmembrane and cytosolic domains are depicted along the protein model ( $97.1 \%$ of the CSMD1 protein is extracellular). b RNA expression of mouse Csmd1 in sexually immature whole testes (20 days), sexually mature whole testes (40 days), and whole ovary. RNA-seq of FACS-purified germ cell populations show Csmd1 expression changes during spermatogenesis. Csmd1 RNA is maximally expressed at the spermatid period of development. Boxplot center line = median; hinges $=$ upper and lower quartiles; whiskers $=1.5 \times$ interquartile range. Line chart error bars reflect standard error of the mean . c CSMD1 protein immunofluorescence in developing oocytes (marked by MVH) and surrounding somatic cells. MVH expression decreases, whereas CSMD1 expression increases with follicular development. CSMD1 encircles the oocytes at all follicle stages. Follicles named in each box are marked with white arrows when necessary. Theca cells are indicated by stars. d CSMD1 is maximally expressed at the oocyte surface and extends into the transzonal projections (white arrowheads), which physically connect the germ cell to the surrounding somatic granulosa cells (left). During ovulation the follicle releases the oocyte and regresses to form the corpus luteum (dashed lines). CSMD1 signal is absent. An oocyte is highlighted for comparison (white arrowhead, right). e Immunofluorescence (IF) visualization of CSMD1 (red) in testis seminiferous tubule cross sections ( $x-y$ axis). CSMD1 protein is broadly expressed in germ cells across all stages of spermatogenesis. $\mathrm{MVH}$ is a primordial germ cell marker whose expression peaks early, then steadily decreases during spermatogenesis/oogenesis. CSMD1 is maximally expressed in elongating spermatids (white arrowheads). $\mathbf{f}$ Whole mount testis tubule preparation ( $z$ axis). F480-positive macrophages (green) with characteristic ramified processes occupy the longitudinal interstitial space. CSMD1 (red) is expressed in a hatched pattern which may correspond to the actin cytoskeleton of peritubular myoid cells and Sertoli cells. Four Sertoli cells surrounding a macrophage outlined by dotted lines. All scale bars $=50 \mu \mathrm{m}$. Source data are provided as a Source Data file

genetic variation; (ii) ascertained by orthogonal genotyping platforms; (iii) occupying multiple points along the allele frequency spectrum; and (iv) in multiple populations and cohorts.

Detailed CSMD1 expression patterns in male and female gonads. CSMD1 encodes for an extremely large (>3,000 amino acid) transmembrane protein with a large extracellular portion consisting of alternating CUB and Sushi complement-interacting domains ${ }^{15}$. The protein encoded by CSMD1 is conserved between human and mouse, with $93 \%$ amino acid sequence identity and $100 \%$ domain synteny (Fig. 2a). In humans, CSMD1 is expressed in both male and female gonads (Supplementary Fig. 2), but extremely little is known of its molecular function, particularly in the context of fertility. Thus, we first performed RNA-seq on 
mouse gonads and confirmed that Csmd1 mRNA is expressed in both whole ovaries and whole testes (Fig. 2b, Supplementary Fig. 3), consistent with previous work ${ }^{30,31}$. Next, we detailed patterns of in situ CSMD1 protein expression through the different stages of ovulation using a validated CSMD1 antibody (Supplementary Fig. 4). Throughout oogenesis, CSMD1 shows lower expression in oocytes of early follicles (i.e. primordial and primary follicles) and higher expression in oocytes of late follicles (i.e. secondary, tertiary, and pre-ovulatory follicles; Fig. 2c). Supporting theca cells, which circumscribe the mature follicles, are also CSMD1-positive (Fig. 2c, asterisk). The post-ovulatory corpus luteum shows no specific CSMD1 expression (Fig. 2d). Female oocytes require substantial physical interaction with surrounding somatic cells ${ }^{32}$. At high magnification, CSMD1 is expressed along transzonal projections which connect the somatic granulosa cells to the oocyte membrane (Fig. 2d).

In the testes, Csmd 1 mRNA is minimally expressed at 20 days and more robustly expressed at 40 days of age which coincides with the onset of sexual maturity. Mammalian testes demonstrate exceptional transcriptional complexity, owing to the highly coordinated spatial and temporal synchronization required for successful spermatogenesis throughout male adult life ${ }^{30}$. Therefore, to capture a more detailed transcriptional portrait of Csmd1 in testes, we purified germ cell subpopulations using established FACS protocols (see Methods section; Supplementary Fig. 3a). Subsequent RNA-seq of purified germ cells reveals low levels of Csmd1 expression in spermatogonia and primary spermatocytes and peak expression in secondary spermatocytes and spermatids (Fig. 2b; Supplementary Fig. 3).

We also performed immunofluorescence on seminiferous tubule cross-sections which show that CSMD1 protein is expressed at the germ cell membrane across multiple aspects of spermatogenesis, including at the interface of elongated spermatids and somatic Sertoli cells, but is absent from spermatozoa, recapitulating mRNA expression patterns (Fig. 2e; Supplementary Fig. 3). While tubule cross-sections effectively capture information from the lumenal stages of spermatogenesis, much of the spatial variance of basal germ cells, Sertoli cells, and interstitial cells (e.g. macrophages) occurs along the longitudinal axis ${ }^{33}$. Therefore, we also performed immunofluorescence on wholemount longitudinal preparations of individual seminiferous tubules. CSMD1 is expressed in a hatched pattern which is reminiscent of the actin bundles found at the Sertoli-Sertoli blood-testis barrier and the Sertoli-spermatid interface ${ }^{34}$ (Fig. 2f).

Csmd1 KO causes fertility phenotypes across multiple tissues. Association studies in humans are effective in identifying candidates for disease risk. However, owing to the extensive linkage disequilibrium structure in the human genome, even statistically robust and reproducible associations may implicate linked but biologically non-causal loci (sometimes up to megabase scales) ${ }^{35}$. In order to confirm a role for CSMD1 in mammalian reproduction, we obtained a previously described mouse strain with a knockout of the first exon of Csmd1 (refs. ${ }^{31,36}$ ) (Supplementary Fig. 4). RNA-seq of knockout females demonstrated robust loss of Csmd1 expression in the ovaries. In testes, we observe a more complex perturbation of expression, with absent or highly reduced expression of exons 1-57, but upregulation of exons 58-70. Finally, we cross-referenced multiple expression modalities (RNA-seq, CSMD1 immunofluorescence, $\beta$-Gal immunofluorescence, and Xgal staining; Fig. 2; Supplementary Fig. 4) and found multiple lines of evidence corroborating expected CSMD1 spatial patterns in wildtype tissues. We also identified nonspecific signal in KO tissues, in particular in our CSMD1 immunofluorescence assay (Supplementary Fig. 4c).
We generated a colony of Csmd1 wildtype, heterozygous, and knockout mice and observed the effect of genotype on fertility, gonadal morphology and function in male and female mice. In males, Csmd1 knockout leads to variable, but statistically significant, defects across multiple dimensions of gonad function and fertility. First, we observed an increase in infertility rate among knockout males (30\%) compared to wildtype (4.5\%) (linear regression $p$-value $=4 \times 10^{-4}$, Fig. 3a). Second, knockout males show on average a $25 \%$ reduction in the daily sperm production compared to controls (Wald test $p$-value $=0.029$, Fig. 3b). Third, a subset of Csmd1 knockout males suffer from profound anatomic and histopathologic derangement of the testes (Fig. 3c-e; Supplementary Fig. 5). Remarkably, the most severe examples of germ cell elimination (Sertoli cell-only syndrome), could be observed as early as 34 days of age (Fig. 3c; Supplementary Fig. 5). This time point corresponds to the approximate onset of male sexual maturity and the emergence of the spermatid germ cells, where Csmd1 is maximally expressed. Neither knockout nor wildtype testes showed evidence of derangement prior to sexual maturity (Supplementary Fig. 5c). Both histologic severity (none, mild, and severe) and age of onset (postnatal day 34 through day 300) were highly variable between individuals. In fact, different foci within the same testis of Csmd1 knockout mice often simultaneously present different degrees of seminiferous tubule degeneration (Fig. 3d). Finally, significantly fewer germ cells express the male germ cell antigen TRA98 (Poisson regression $p$-value $<2 \times 10^{-16}$; Supplementary Fig. $5 \mathrm{~d}$ ), in both atrophic and normal tubules, suggesting that knockout testes suffer from expression perturbations in addition to, or perhaps presaging, loss of germ cells and frank degeneration. Together, these observations indicate that the Csmdl knockout mutation is not fully penetrant and may be influenced by environmental and/or stochastic events. However, even after accounting for age covariates, testes derangement status segregates significantly with Csmd1 genotype (multivariate ANOVA $p$ value $=7.69 \times 10^{-3}$; see Methods section; Fig. 3e). Lastly, we performed serial backcrossing for 9 generations on a subset of mice to validate the effect of the Csmd1 null allele on an approximately constant genetic background (see Methods section). We recapitulated the degeneration phenotype in these backcrossed male knockouts (Supplementary Fig. 5e), indicating that Csmd1 genotype status - not genetic background - drives this signal of degeneration.

Detailed histological analysis of 50 knockout testes revealed heterogenous and distinct classes of degeneration in Csmd1 $1^{-/-}$ testes (Fig. 3d). Spermatogenesis begins to become disorganized, especially at the late steps of spermiogenesis, with failure of spermiation, fewer numbers of elongating spermatids in the lumen, and ectopic mixing of spermatid steps in stages IX-XII. This is followed by the sloughing of all types of germ cells into the lumen; remaining germ cells can be observed in abnormal tubules that appear to be missing one or more waves of spermatogenesis, and these eventually resolve as Sertoli cell-only tubules. Sloughed germ cells can be seen downstream in the epididymis, and, occasionally they obstruct the downstream rete testis leading to dilation of the upstream tubules.

Three non-exclusive scenarios may account for the net loss of germ cells in Csmd1 knockouts: (i) increased germ cell death; (ii) failure of germ cell proliferation/maturation; and (iii) increased phagocytic clearance of germ cells. We did not observe obvious differences in the abundance of apoptotic germ cells apparent by $\mathrm{H} \& \mathrm{E}$ staining. We excluded systemic endocrine defects that would be observed in the case of failure of the hypothalamus or pituitary (Supplementary Fig. 6). We also did not observe any developmental stage-specific accumulation or depletion of germ cells as characterized in maturation arrest/failure of meiosis ${ }^{37,38}$. 
a

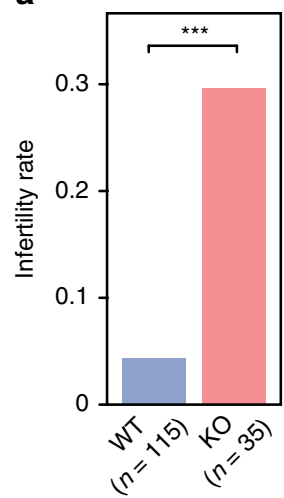

d

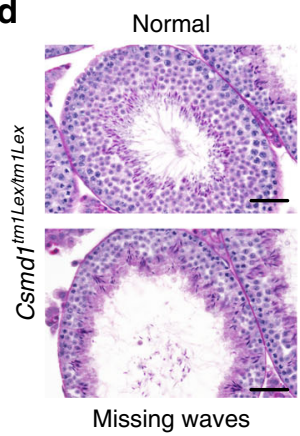

b

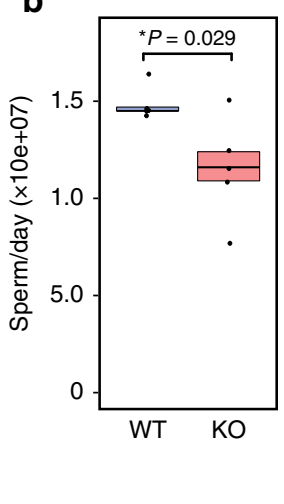

Disorganized

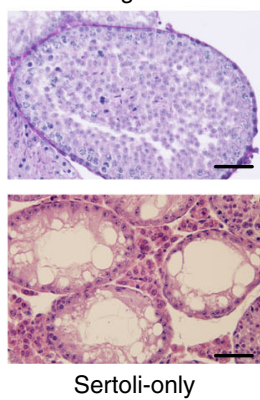

C

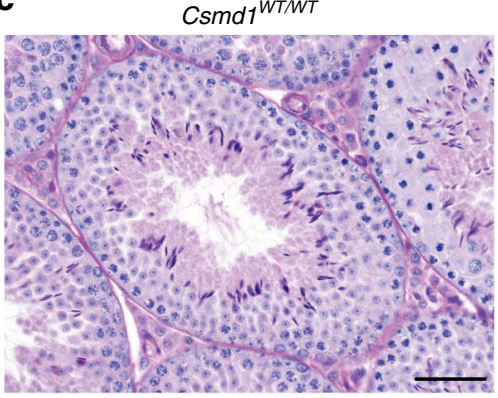

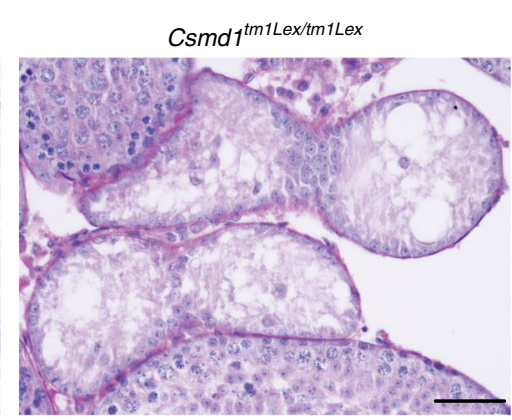

e

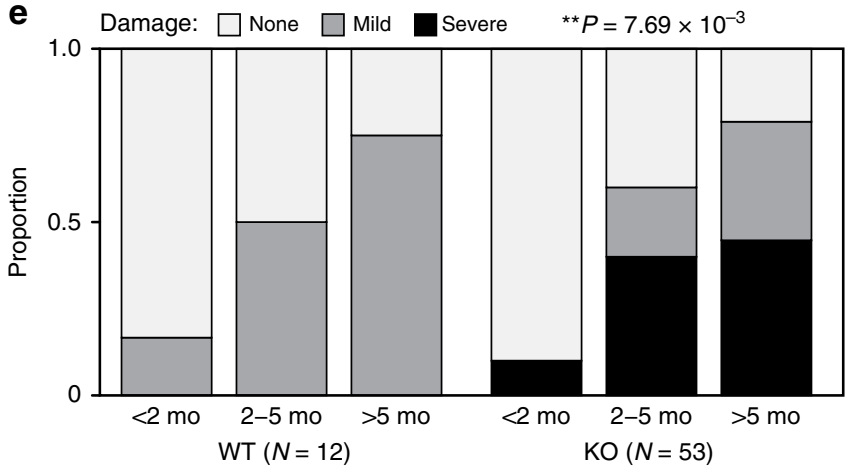

Fig. 3 Variable reproductive defects in Csmd1 KO males. a Barplot depicting proportions of infertile Csmd1 wildtype (WT) vs. knockout (KO) males. KO males showed significantly higher rate of infertility (relative risk $=6.8$, linear regression $p$-value $=4 \times 10^{-4}$ ). $\mathbf{b}$ Daily sperm production is $25 \%$ lower in knockout testes after controlling for age (Wald test $p$-value $=0.029)$. Center line $=$ median; hinges $=$ upper and lower quartiles. $\mathbf{c}$ Seminiferous tubule histology of wildtype vs. knockout littermates at postnatal day 34. The majority of knockout tubules in this sample contain no germ cells (Sertoli-only). d Qualitative classes of progressive morphologic degeneration. Seminiferous tubules from Csmd1 knockout males showing normal morphology (Normal); loss of spatiotemporal structure, but retained germ cells in all phases of spermatogenesis (Disorganized); loss of early germ cells into the tubule lumen (Sloughing); loss of all germ cells except late-step spermatids (Missing waves); and loss of all germ cells, leaving a signature of vacuolization (Sertoli-only). Sloughed germ cells from upstream tubules are occasionally captured in the downstream epididymis (Ectopic germ cells). Csmd1 knockout males can display multiple classes of degeneration even within the same testis. Like the histological phenotypes, fertility status varies greatly among knockout individuals, with some males becoming infertile within weeks, and others within months. e Quantification of the degeneration phenotype. Testis sections from Csmd 1 wildtype and knockout animals were assigned one of three possible histological degeneration scores: None $=0$, Mild $=1$, or Severe $=2$ (see Methods section). The stacked barplots depict the proportion of damaged tubules among wildtypes and knockouts, stratified by age group. All proportions sum to 1. Damage severity segregates significantly with genotype after controlling for age (ANOVA $p$-value $=7.69 \times 10^{-3}$ ). All scale bars $=50 \mu \mathrm{m}$. Source data are provided as a Source Data file

Finally, we did not observe significant differences in the proliferation marker PCNA levels between Csmd1 wildtype (9.4\%) and knockout (7.0\%) testes.

Complement C3 deposition is increased on Csmd1 KO testes. FACS-purification and RNA-seq of wildtype male germ cells show that $C 3$ and Csmd1 mRNA expression are negatively correlated throughout spermatogenesis (Fig. 4a); in contrast to Csmd1, C3 mRNA is most highly expressed in spermatogonia and decreases precipitously in subsequent stages. In wildtype testes, we observed abundant signal of both $\mathrm{C} 3$ protein and macrophage marker F4/80 in the interstitial space between seminiferous tubules, but never across the blood-testis barrier (Fig. 4b). Next, we estimated macrophage abundance and C3 protein deposition in Csmd1 wildtype vs. knockout testes (Fig. 4c; Supplementary Fig. 7). The percentage of C3-positive deposition is significantly higher in Csmd 1 knockout testes than in wildtype $\left(\overline{\mathrm{x}}_{\mathrm{wt}}=1.7 \% ; \overline{\mathrm{x}}_{\mathrm{ko}}=6.6 \%\right.$; ANOVA $p$-value $\left.=7.7 \times 10^{-4}\right)$. C3 is a secreted, circulating protein whose mRNA is expressed by multiple cell types - including the germ cells themselves. Therefore, we also measured C3 mRNA expression in Csmd 1 wildtype versus knockout purified male germ cells (Fig. 4d). C3 mRNA expression does not differ between wildtype and knockout germ cells of any type, indicating that the germ cells are not necessarily the source of the increased C3 protein deposition.

Csmd1 KO ovaries are reduced in quality. The observed rate of infertility in knockout females $(3 / 37,8.1 \%)$ was not significantly higher compared to controls $(5 / 115,4.3 \%$, Fig. $5 a)$. The female infertility phenotype we ascertained in human does not likely represent congenital infertility, but instead adult-onset early menopause, which is difficult to model in mice ${ }^{39}$. Thus, we searched for more subtle gonadal defects associated with Csmd1 genotype. We estimated female time to pregnancy based on retrospective husbandry records. From these records, we generated a well-calibrated null distribution of time to conception in females of all genotypes which demonstrates distinct periodicity lasting 4-5 days, recapitulating the periodicity of the mouse female estrous cycle (Fig. 5b). Next, we stratified our population by maternal genotype. For wildtype mothers, the bulk of conceptions occurred within the first estrous cycle as expected ${ }^{40}$, whereas most Csmd 1 knockout mothers required a time equivalent to two or more cycles to achieve pregnancy $\left(\beta_{\mathrm{GT}}=10.4\right.$; linear regression $p$-value $=0.012$ ). A small minority of knockout females required many cycles to achieve pregnancy ( $>60$ days). Circulating gonadotropin levels did not differ between wildtype and 


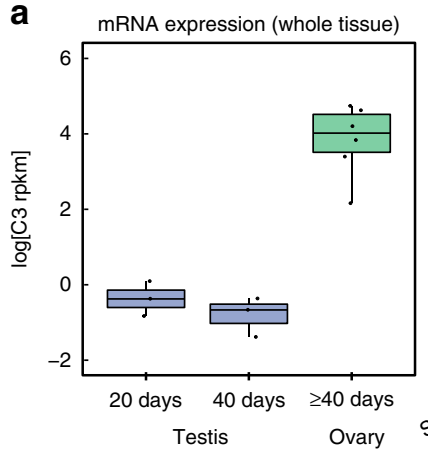

C

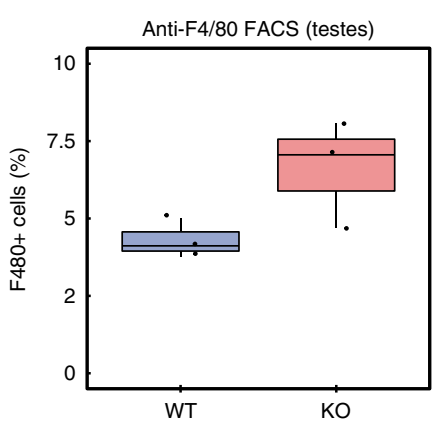

mRNA expression (germ cells)

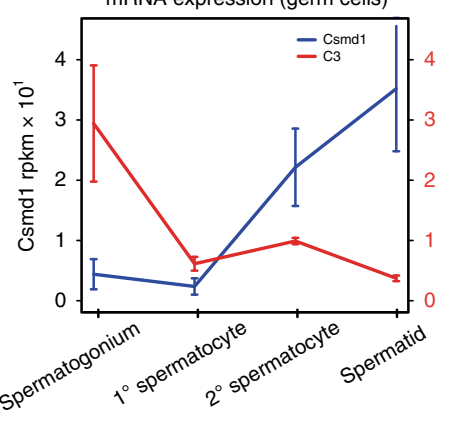

b

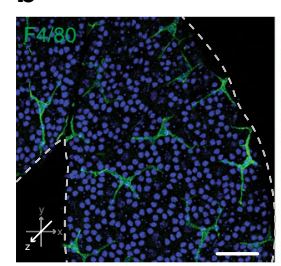

Seminiferous tubules

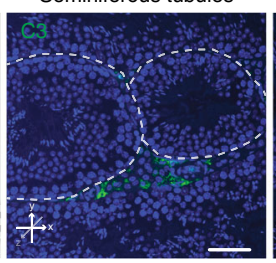

Epididymis

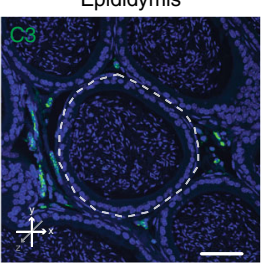

d

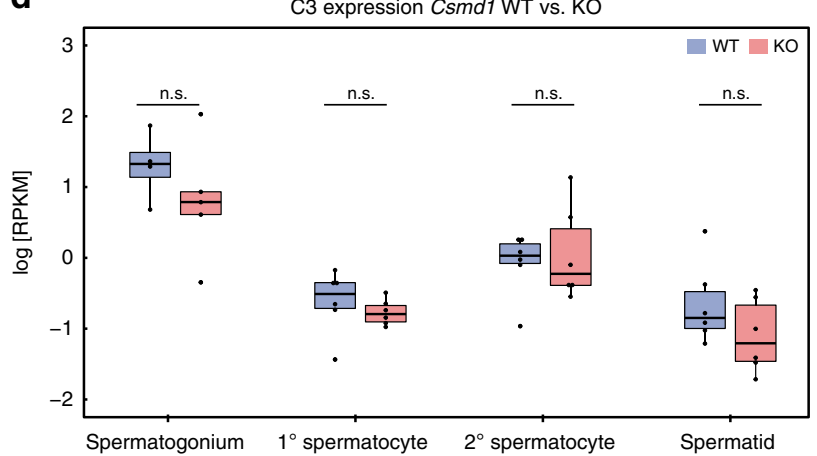

Fig. 4 Normal and aberrant complement C3 localization in Csmd1 wildtype versus knockout testes. a C3 mRNA expression in sexually immature whole testes, sexually mature whole testes, and whole ovary. RNA-seq of FACS-purified germ cell populations shows C3 expression decreases throughout spermatogenesis. Csmd1 expression data from Fig. 2 are rescaled and superimposed for ease of comparison. Line chart error bars reflect standard error of the mean. $\mathbf{b}$ Complement and macrophages are confined to the interstitial compartment in normal tubules. F4/80 immunofluorescence of whole mount tubule preparations shows abundance of macrophages along the interstitium (left). C3 immunofluorescence of tubule cross-sections shows expression in the interstitium, but not within the lumenal compartment (middle). Cross-section of downstream epididymis shows continued exclusion of $\mathrm{C} 3$ from the lumenal compartment (right). Individual tubules are circumscribed by dashed lines. Scale bars $=50 \mu \mathrm{m}$. c Boxplots depicting F4/80 abundance and C3 protein deposition in FACS-sorted Csmd1 wildtype vs. knockout testes. Both F4/80 and C3 are increased in Csmd1 knockout testes, though only significant for C3 (ANOVA $p$-value $=7.7 \times 10^{-4}$ ). d RNA-seq of Csmd1 wildtype vs. knockout germ cell populations shows no significant difference in C3 mRNA expression (two-tailed $t$-test; $p$-value $>0.05$ ). For all boxplots, center line $=$ median; hinges $=$ upper and lower quartiles; whiskers $=1.5 \times$ interquartile range. Source data are provided as a Source Data file

knockouts after controlling for estrous stage, suggesting that this reduction in mating success was not secondary to impaired hormonal input (Supplementary Fig. 6). Csmd1 knockout females had significantly smaller ovaries by mass when controlling for age, (quadratic regression $p$-value $=8.1 \times 10^{-3}$; Fig. $5 c$; Table 1 ), and knockout females showed significantly more atretic follicles and fewer normal pre-ovulatory follicles at necropsy (Hotelling $t$-test $p$-value $=3.5 \times 10^{-3}$; Fig. $\left.5 \mathrm{~d}\right)$. In a subset of female knockout ovaries, we observed inflammatory changes associated with infiltration of lipid-engorged macrophages (foam cells) and occasional ovarian cysts (Fig. 5e, f). Altogether, we find that Csmd1 knockout females display ovarian defects that are normally associated with progressive wildtype aging (i.e. smaller ovary size ${ }^{41}$, increase in atretic follicles ${ }^{42}$, and enrichment of foam cell macrophages ${ }^{43}$ ), but at significantly accelerated rates and higher proportions in knockouts.

C3 is abundant in follicles and colocalizes with CSMD1. In wildtype ovaries, C3 is localized to the oocyte surface in normal developing follicles, colocalized with CSMD1, and then observed to be diffused in large amounts throughout the corpus luteum (Fig. 6), which does not express CSMD1. Macrophages are a prominent cell type in the ovarian stroma but are excluded from early stage follicles; they later invade corpora lutea and degrading follicles ${ }^{44}$. Furthermore, C3 colocalizes with macrophages in the corpus luteum, atretic follicles, and among developing follicles to variable degrees. C3 is also abundant within the early follicular antrum, suggesting that C3 may be important for remodeling the connections between granulosa cells during antrum formation (Fig. 6b). Previous work has shown that activated C3 is present in human antral follicular fluid at levels comparable to sera ${ }^{45}$.

Csmd1 KO mothers have impaired mammary branching. Interestingly, while knockout females achieved fewer pregnancies per estrous cycle, the average number of offspring born per successful pregnancy did not differ significantly between wildtype and knockout mothers $\left(\overline{\mathrm{x}}_{\mathrm{wt}}=6.6\right.$ (95\% CI $\left.[5.4-7.8]\right) ; \overline{\mathrm{x}}_{\mathrm{ko}}=6.9$ (95\% CI [5.7-8.1]); Table 1). However, pups borne of Csmd1 knockout mothers suffered from significantly higher mortality rates during the neonatal period (1-10 days) when compared to wildtype/heterozygous mothers (\% mortality ${ }_{\mathrm{wT}+\text { het }}=10.5 \%$ (95\% CI [3.6-17.5\%]); \% mortality KO $=50.0 \%$ (95\% CI [30.0 $-70.0 \%]$ ); Poisson regression $p$-value $=7.93 \times 10^{-7}$; Fig. 7a). We performed necropsy on expired offspring which revealed an absence of milk spots, suggesting death by starvation. Because neonatal mortality segregated with maternal genotype but not offspring genotype or paternal genotype, we hypothesized that this increase in mortality was caused by nursing defects in Csmd1-deficient mothers. CSMD1 is expressed in the normal mammary gland through the adult life cycle of wildtype females 
a

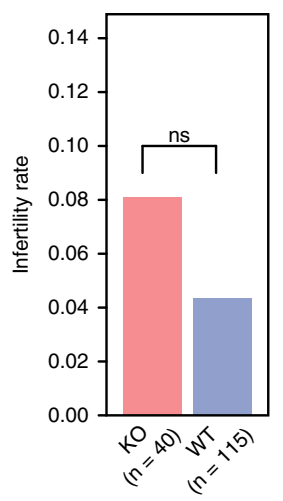

e

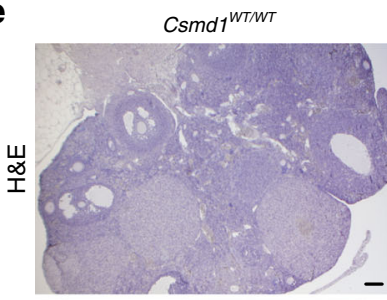

b

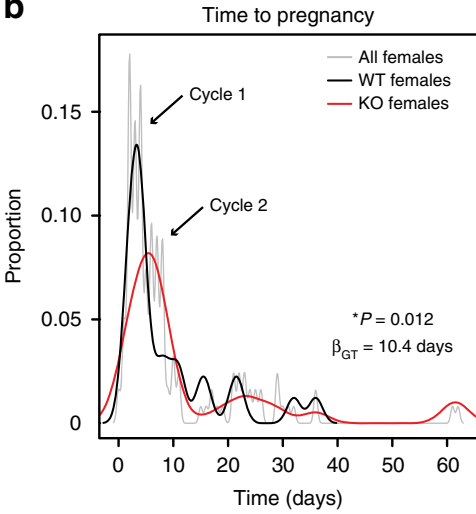

C

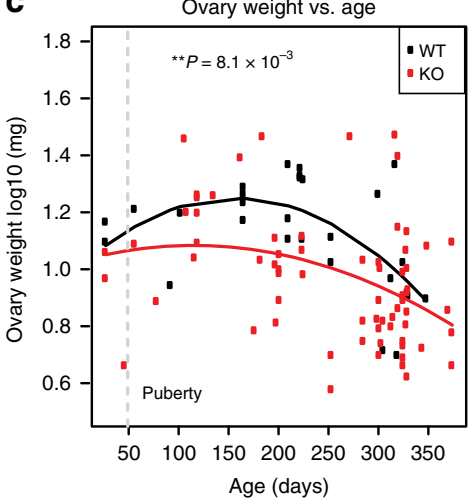

f

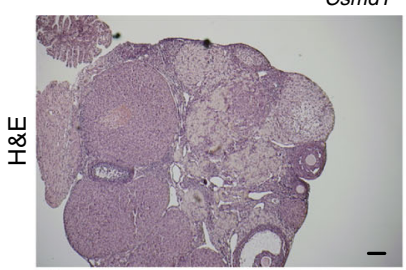

d

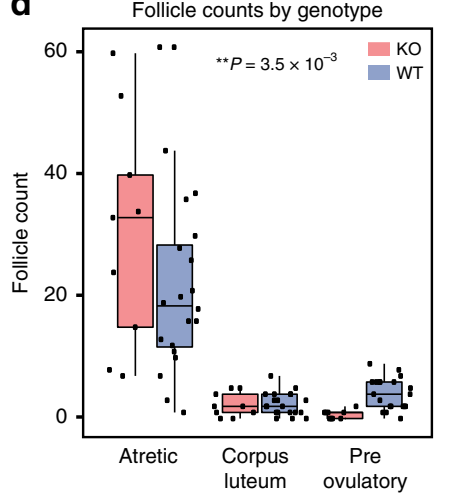

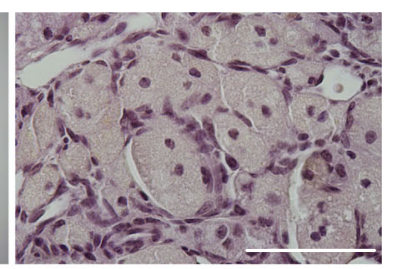
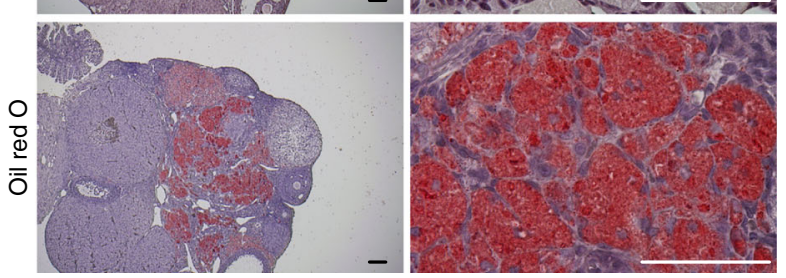

Fig. 5 Csmd 1 knockout ovaries show reduced morphologic quality and reproductive performance. a Barplot depicting proportions of infertile Csmd1 wildtype (WT) vs. knockout (KO) females. In the population sampled ( $n=115 \mathrm{WT}+40 \mathrm{KO})$, Csmd1 genotype does not significantly predict infertility status in females (relative risk $=1.9$, linear regression $p$-value $>0.05$ ). b Probability density plot depicting female mating success over time, stratified by maternal genotype (black = wildtype females; red = knockout females; gray=all females). The distribution is periodic, recapitulating the periodicity of the mouse female estrous cycle. Knockout females require significantly more cycles to achieve pregnancy $\left(\beta_{\mathrm{GT}}=10.4\right.$, linear regression $p$-value $\left.=0.01\right)$, with a small subset of females never achieving pregnancy. All statistical models account for confounders such as age, body weight, and male factors when appropriate (see Methods section). c Scatterplot depicting ovary weight versus age, stratified by genotype (black= wildtype females; red = knockout females). The fitted polynomial models are depicted as lines with color corresponding to genotype. Knockout females have significantly smaller ovaries than wildtype when controlling for age (quadratic regression $p$-value $=8.1 \times 10^{-3}$ ). Gray hashed line demarcates rough onset of puberty in females. $\mathbf{d}$ Composition of follicles differs significantly between wildtype and knockout female ovaries, with significantly fewer pre-ovulatory follicles and significantly more atretic follicles in knockout (Hotelling $t$-test $p$-value $=3.5 \times 10^{-3}$ ). Center line $=$ median; hinges $=$ upper and lower quartiles; whiskers $=1.5 \times$ interquartile range e Ovarian histology in a wildtype versus knockout female. Knockout ovaries were consistently enriched for foam cell macrophages, confirmed by Oil Red $\mathrm{O}$ stain in adjacent sections. We occasionally observed ovarian cysts in knockout females (asterisk). f Ovary from 336-day-old knockout showing extensive involvement of foam cells occupying $40 \%$ of the tissue (left). High magnification of ovary section from same animal; top shows multinucleated appearance of foam cells, bottom is Oil Red O stain of adjacent section (right). All scale bars $=100 \mu \mathrm{m}$. Source data are provided as a Source Data file

\begin{tabular}{|c|c|c|}
\hline & WT & ко \\
\hline Ovary weight & $0.057[0.024-0.090]$ & $0.028[0.017-0.039]$ \\
\hline Mammary weight & $0.48[0.35-0.61]$ & $0.37[0.26-0.48]$ \\
\hline Testis weight & $0.29[0.24-0.34]$ & $0.27[0.25-0.29]$ \\
\hline Maternal litter size & $6.6[5.4-7.8]$ & $6.9[5.7-8.1]$ \\
\hline Paternal litter size & $5.4[3.4-7.4]$ & $7.4[6.6-8.2]$ \\
\hline
\end{tabular}

Mean values $+/-1.96$ standard errors of the mean. All weights are the sum of bilateral measurements per animal in grams. Litter size measurements are stratified by maternal and paternal genotype and do not include individuals that failed to conceive (i.e. they only include non-zero litter sizes) on both lumenal epithelial cells and myoepithelial cells of the mammary ducts, and on numerous stromal cells (Fig. 7b, c). Mammary glands from knockout females showed reduced density of the epithelial branching network during mid-pregnancy and post-nursing (Fig. 7d). Quantification of duct morphology in nulliparous wild type and knockout animals shows a significant reduction of lateral branching prior to pregnancy (Fig. 7e, f). Finally, we measured C3 and CSMD1 expression patterns in wildtype mammary glands (Fig. $7 \mathrm{~g}$ ). As early as puberty, C3 can be seen in high levels within the mammary duct lumen of virgin animals. C3 is also expressed within vesicles of specific subsets of CSMD1-positive stromal cells (Fig. 7g).

Csmd1 ${ }^{-/-} \mathrm{C3}^{-/-}$double $\mathrm{KO}$ males and females are infertile. Based on previous findings that CSMD1 is a negative regulator of $\mathrm{C} 3$, we predicted that removal of $\mathrm{C} 3$ would partially or completely 
a

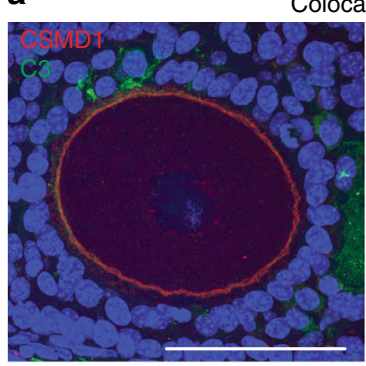

Colocalization

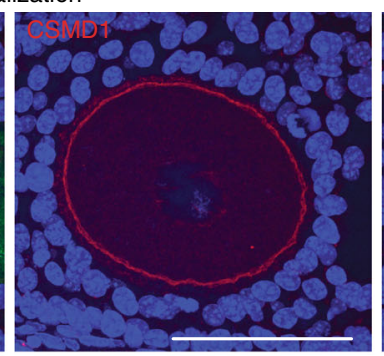

b

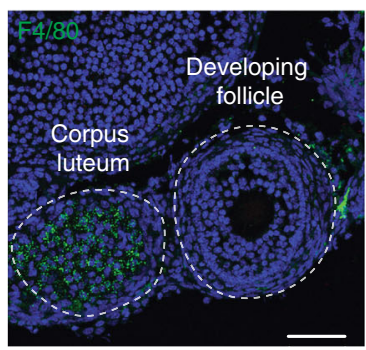

Developing follicle

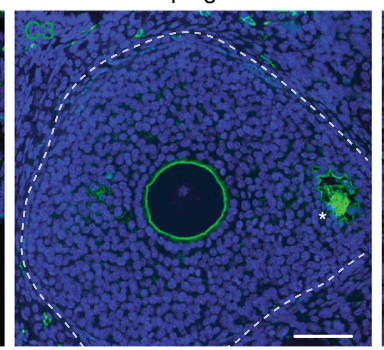

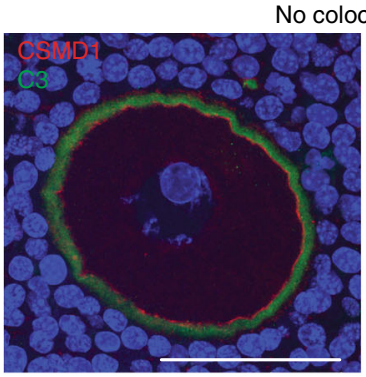

Atretic follicles

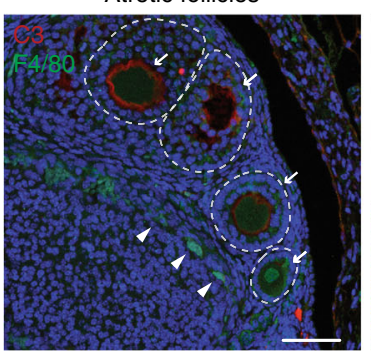

No colocalization

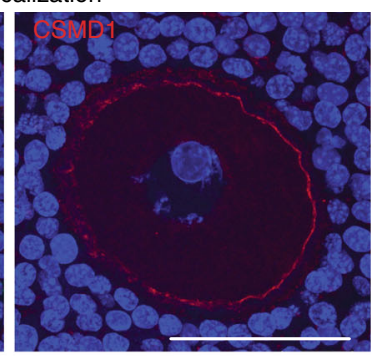

Corpus luteum

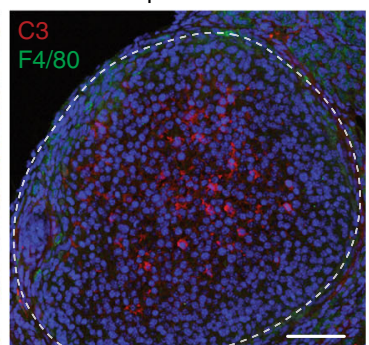

Fig. 6 C3 and CSMD1 protein colocalize at the oocyte surface. a Spatial relationships between C3 and CSMD1 in developing follicles. C3 and CSMD1 immunofluorescence of oocytes in developing follicles. In most follicles, C3 and CSMD1 colocalize at the oocyte plasma membrane with overlapping signal (Colocalization, left panels). Occasionally the two signals separate and C3 is localized in a tight but distinct ring outside of CSMD1 (no colocalization, right panels). b Spatial relationships between C3 and F4/80-positive macrophages in developing, atretic, and post-ovulatory follicles. F4/80

immunofluorescence of adjacent follicles shows positive macrophage signal in corpus luteum, but not within developing follicles. C3 is localized to the oocyte membrane, as well as in the follicular fluid of the developing antrum (asterisk). Atretic follicles at different stages of degeneration (white arrows) show varying levels of C3 and F4/80 expression. F4/80 can also be seen in a punctuated pattern along the stroma and thecal layers (white arrowheads). C3 is also present in corpus luteum. Individual follicles are circumscribed by dashed lines. All scale bars $=50 \mu \mathrm{m}$. Source data are provided as a Source Data file

alleviate the morphological degeneration and fertility defects observed in Csmd1 knockout mice. To test this prediction, we generated colonies of $C 3^{-1-}$ single $\mathrm{KO}$ mice and $\mathrm{C} 3^{-1-}$ Csmd $1^{-1-}$ double $\mathrm{KO}$ mice. We did not observe any severely deranged $C 3^{-1-}$ testes or ovaries (Supplementary Fig. 8). Surprisingly, we found no evidence of rescue of the Csmd1 ${ }^{-/-}$single $\mathrm{KO}$ phenotype in double KO males or females (Supplementary Fig. 9). Instead, we observed an unmasked phenotype of more severe histological degeneration in all DKO females, characterized by far more extensive invasion of foam cell macrophages, extensive pyknosis, and deformed follicles. We also observed loss of cellularity in the mucosal layer of the oviduct (Supplementary Fig. 9b). We monitored the fertility of 19 DKOs (10 males and 9 females) and, of these, only $4(21 \%)$ produced progeny after at least 3-7 months of mating (3 males and 1 female; Supplementary Fig. 9, Supplementary Table 3). These extreme phenotypes are not observed in Csmd1 or $C 3$ single $\mathrm{KOs}^{46}$, suggesting that the combined effect of Csmd1 and C3 on fertility is synergistic.

\section{Discussion}

We used a rare variant association approach to search for genes that modulate both male and female gonadal function, and identified the complement regulator CSMD1 to be reproducibly associated across multiple patient cohorts and different classes of genetic variation. Our study was powered to capture rare variants of mild to moderate effect - that is, mutations that significantly increase risk of developing infertility, but still potentially segregating in the population. Conversely, our study was poorly powered to identify extremely rare/de novo, highly penetrant infertility mutations (e.g. TEX11 (ref. ${ }^{38}$ ), SYCE1 (refs. ${ }^{38,47}$ ), $\left.S P I D R^{48}\right)$. We found that multiple types of genetic perturbations of CSMD1 associate with infertility traits in humans, most notably deletions within introns $1-3$ of the gene. Intronic deletions can affect gene function by altering splicing, RNA stability, and transcriptional regulation, and recent work has shown that such deletions tend to reduce transcript levels when they perturb expression of their host gene, although upregulation is also possible $^{49}$. All infertility-associated deletions found in this study fall within $500 \mathrm{~kb}$ of 3 independent GWAS peaks for age at menarche. In the GTEx atlas, we found that only two tissues show significant CSMD1 expression-associated quantitative trait loci (eQTL): thyroid and testis ${ }^{50}$ (Supplementary Fig. 10). Although the CSMD1 gene body spans over $2 \mathrm{Mb}$ of the genome, all of these eQTL are clustered within a $390 \mathrm{~kb}$ window spanning introns 1 and 2. The 3 noncoding GWAS SNPs from Day et al. ${ }^{28}$ are not in appreciable LD with the GTEx eQTL. We also screened $\mathrm{ENCODE}^{51}$ for functional annotations in this region. Functional summaries (e.g. chromHMM output) are not yet available for testis, ovary or mammary gland; instead we used functional summaries derived from 6 human cell lines and identified 4 consensus CTCF sites within the region (Supplementary Fig. 10). We conclude that CSMD1 introns 1 and 2 likely harbor functional elements that can influence gene expression in testis, but additional functional data are needed to map these elements, as well as potential elements in ovary and mammary gland.

Furthermore, we found that rare protein-coding SNVs within the CUB domains of CSMD1 are significantly associated with age at menopause. According to the Residual Variation Intolerance Score (RVIS) only $0.169 \%$ of genes in the genome are more intolerant to protein coding changes than CSMD1 (ref. ${ }^{52}$ ). Likewise, CSMD1 is massively depleted for rare loss-of-function (LoF) variation in the gnomAD reference database ${ }^{53}$, with a $\mathrm{pLI}$ score of 1.0. This depletion of LoF variation is especially striking 
a

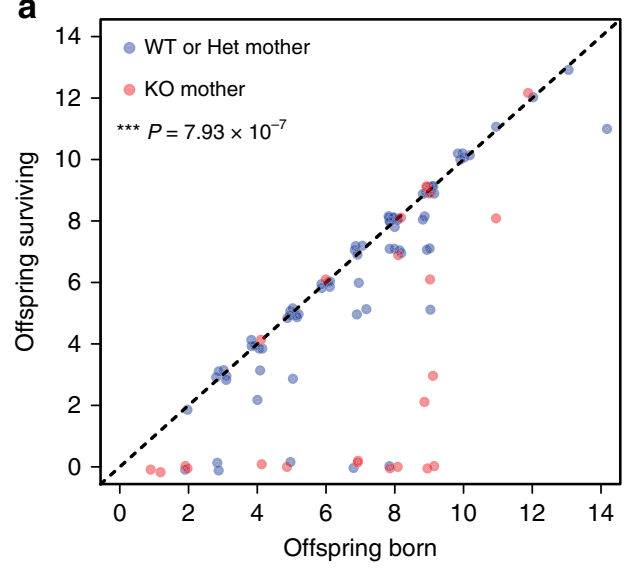

b
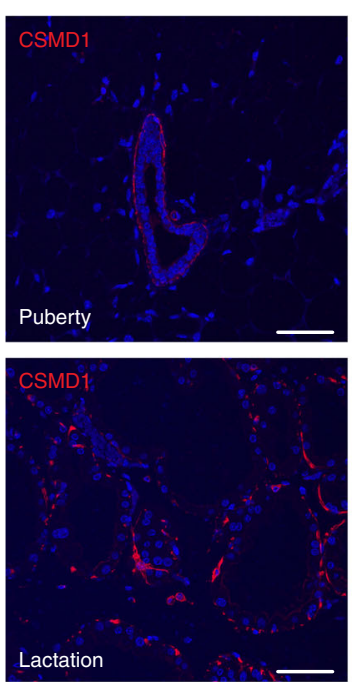

c
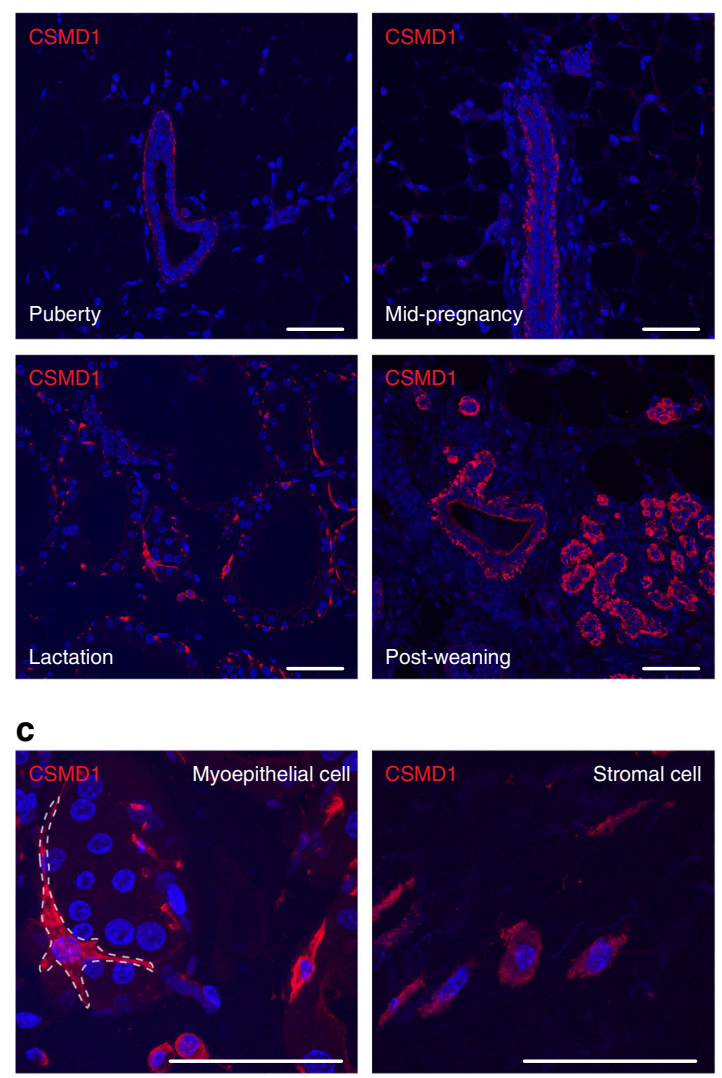

g
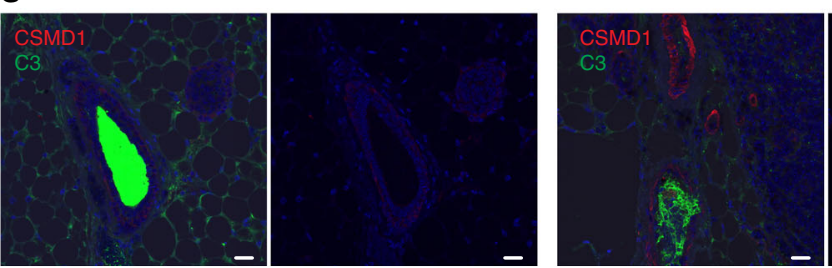

given the local increase in CSMD1 de novo SNV mutation rate ${ }^{54,55}$, which is also corroborated by the substantial enrichment of rare synonymous variation in CSMD1 (ref. ${ }^{53}$ ). Altogether we favor a LoF effect of CSMD1 mutations in human primary gonadal dysfunction.

How do our mouse studies relate to the human genetic findings and what are their limitations? We characterized a Csmd1 genetic knockout construct that was not strictly identical to any of the

d

e

f
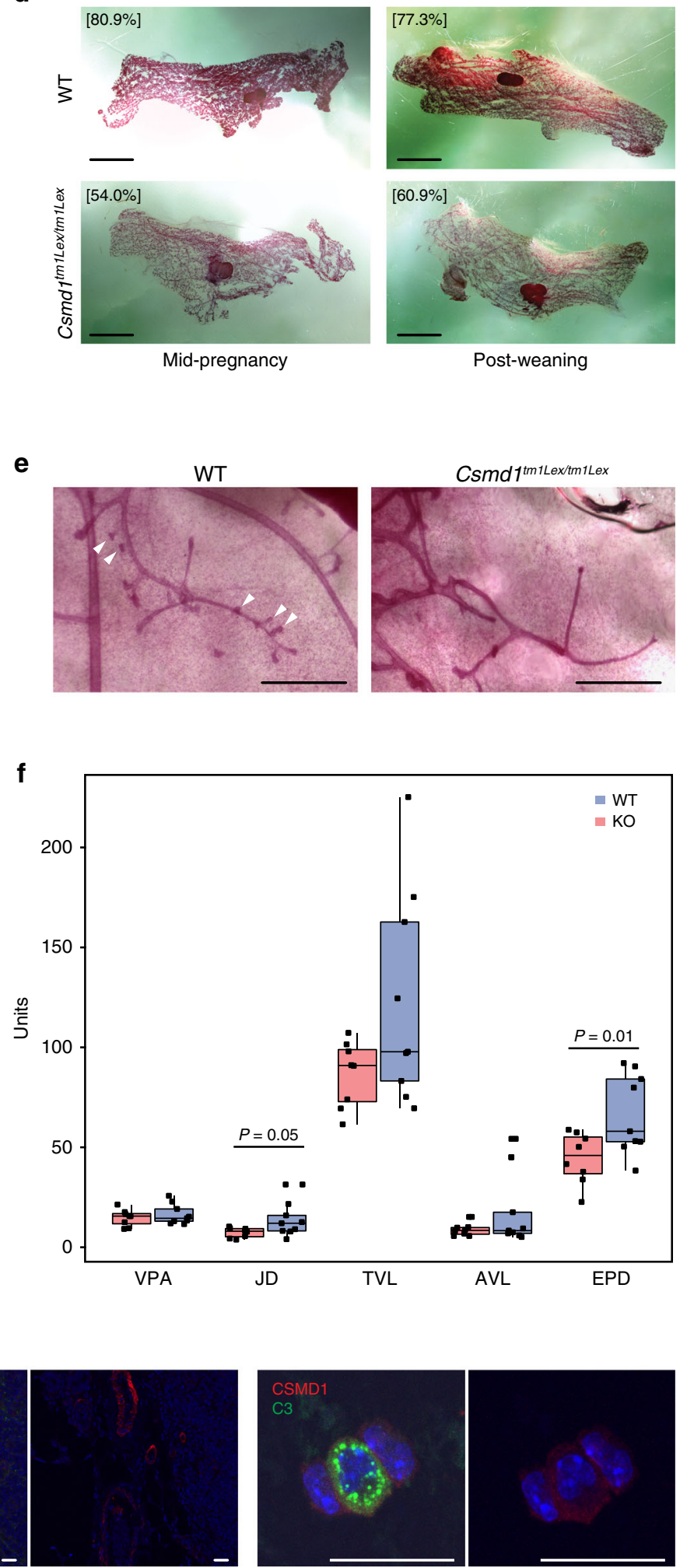

rare patient mutations found in our study. Further complicating our interpretation of the mouse data is our finding that Csmd1 $\mathrm{KO}$ gonads display nonspecific and/or residual expression signal; a phenomenon previously observed in Csmd1 $\mathrm{KO}$ brain $^{36}$. Given the exceptional intolerance of CSMD1 to LoF variation in humans, we hypothesize that incomplete Csmd1 loss-of-function is buffered by functional redundancy ${ }^{56}$ (e.g. from Csmd2 and Csmd3 $)^{57}$, and that complete loss-of-function of Csmd1 would 
Fig. 7 Csmd1 $\mathrm{KO}$ mothers have increased offspring mortality related to maternal nursing defects. a Scatterplot depicting number of pups surviving the neonatal period versus live births, stratified by maternal Csmd1 genotype. Points that lie along the dashed line (slope $=1$ ) represent litters with no neonatal deaths. Maternal Csmd1 genotype is significantly associated with surviving litter size ( $10.5 \%$ vs. $50.0 \%$ survival; Poisson regression $p$-value $=7.93 \times 10^{-7}$ ). Points deviate slightly from whole numbers for ease of visualization. b Immunofluorescence shows CSMD1 expression in bifurcating mammary ducts and bulbous terminal end buds. CSMD1 is expressed on both lumenal epithelial cells and myoepithelial cells throughout different stages of adult life. Expression is lowest at puberty and increases during pregnancy, with the highest intensity during involution. Scale bars $=50 \mu \mathrm{m}$. c Higher magnification image of CSMD1 expression in a myoepithelial cell (dashed lines, left) surrounding an alveolus during lactation and stromal cells (right). Scale bars $=50 \mu \mathrm{m}$.

d Whole-mount mammary glands of wildtype and knockout littermates show reduced epithelial network density (square brackets) in knockouts. Scale bars $=5 \mathrm{~mm}$. e Knockout ducts demonstrate reduced number lateral branches (arrowheads). Scale bars $=500 \mu \mathrm{m}$. $\mathbf{f}$ Image analysis in age-matched wildtype vs. knockout nongravid nulliparous adult mammary ducts. Knockout females have significantly reduced number of branch points along the ducts $(J D$, two-tailed $t$-test $p$-value $<0.05)$ and reduced number of end segments $(E P D$, two-tailed $t$-test $p$-value $<0.01)$. VPA $=$ percent area occupied by ducts. $\mathrm{JD}=$ branchpoint density per $\mathrm{mm}$. TVL = sum of Euclidean distances among adjacent branchpoints. $\mathrm{AVL}=$ average Euclidean distance between adjacent branch points. EPD = number of duct end points normalized by total vessel length. Center line = median; hinges = upper and lower quartiles;

whiskers $=1.5 \times$ interquartile range. $\mathbf{g}$ C3 and CSMD1 colocalize in mammary. Immunofluorescence shows abundant C3 signal within mammary ducts with empty lumina (left) and ducts with cells in the lumen (middle). C3 signal is also present in some CSMD1-positive stromal cells (right). Scale bars $=20 \mu \mathrm{m}$. Source data are provided as a Source Data file

lead to much more severe and/or systemic phenotypes in both humans and mice. A complete tissue-specific and/or inducible loss-of-function knockout of Csmd1 could help to better disentangle these potential effects but would be technically prohibitive due to the size of the gene.

Despite these practical limitations, the mouse mutants provided an invaluable and previously undescribed functional link between CSMD1 and reproductive function. Our human samples were ascertained based on pathologic loss of germ cells, and/or reduced reproductive lifespan. We observed heterogeneous gonadal defects corresponding to reduced reproductive lifespan and premature germ cell loss in both male and female Csmd1 $\mathrm{KO}$ mice, and these phenotypes were unexpectedly exacerbated in Csmd1 $1^{-/}, C 3^{-1-}$ double KO mice. While we have neither explicitly nor implicitly defined the mechanism of CSMD1 mutations on gonadal function here, we have constructed a framework that implicates overzealous activation of the complement pathway as a potential contributor to the pathophysiology of the disease, due to the known molecular function of CSMD1 as a complement inhibitor ${ }^{15}$, the spatiotemporal patterns of complement-related proteins and cell types in the gonads, and the excess complement deposition on Csmd1 KO testes.

While complement has a long-appreciated role in innate immunity, it also plays a prominent role in the regulation of self cells during normal postnatal development. A classic example of this phenomenon is complement-mediated synaptic pruning in the developing brain, a process that is under genetic influence and can confer risk for disease when dysregulated ${ }^{20,58}$. Here, we have reported abnormal post-natal development in Csmd1 knockout animals for three additional tissues; we have observed complement and CSMD1 protein expression in all three tissues, and previous work has shown that macrophages are essential for proper development in all three tissues. A parsimonious model to describe the set of defects we observe here is that macrophages (and potentially other phagocytes such as Sertoli cells) regulate and refine developing cells in testis, ovaries, and mammary by controlled deposition of complement onto their cell surface, accompanied by subsequent phagocytosis.

Specifically, in the testis, increased C3 deposition in Csmd1 knockout taken in context of the known complement-inhibitory function of CSMD1 (ref. ${ }^{23}$ ) suggests that overzealous complement-mediated phagocytosis of developing germ cells by macrophages and/or Sertoli cells underlies at least part of the testicular defects. C3 mRNA and protein are much more highly expressed in the interstitial spermatogonial layer, which is physically sequestered from the lumenal aspect of the tubules by the blood-testis barrier; ${ }^{59}$ Conversely, CSMD1 is largely expressed in the adlumenal compartment (Fig. 2e, f). Spermatogonial fate is closely tied to interstitial macrophages ${ }^{33}$, which are abundant in the testis, express factors essential for spermatogonial differentiation, and enter the tubules and phagocytose germ cells during aging and disease ${ }^{60,61}$. Concerted apoptosis of differentiating spermatogonial stem cells is a prominent and quantitatively reproducible feature of normal spermatogenesis ${ }^{62}$. If complement marks some targets of phagocytosis in the testis in an analogous manner to that shown in the brain ${ }^{63}$, ectopic complement activation across the blood-testis barrier may inappropriately activate the apoptotic and phagocytic apparatus in Csmd1 KO testes. Remarkably, TEP1 (a distant ortholog of C3) has been shown to clear apoptotic germ cells in the mosquito testis by this very process ${ }^{64}$

Macrophage activity in the ovary is very carefully regulated in time and space during the estrous cycle. Prior to ovulation, macrophages are excluded from the granulosa layer of follicles of all types - except the atretic follicles, which are invaded by macrophages and do not ovulate ${ }^{65-67}$. After ovulation, macrophages invade the ruptured follicle which undergoes apoptosis/ phagocytic luteolysis, forming the corpus luteum ${ }^{68}$. We showed that CSMD1 is also highly expressed on oocytes of the developing follicle, but not in the corpus luteum (Fig. 2d). CSMD1 loss of function may allow for premature macrophage invasion of the developing follicle, leading to excessive oocyte atresia, fewer ovulations, and reduced probability of pregnancy (Fig. 5).

In addition to the gonads, CSMD1 may also participate in postnatal developmental processes across other tissue systems. We have demonstrated a robust association between neonatal mortality rate and maternal Csmd1 genotype status, with corresponding reduction in the epithelial network of the maternal mammary gland (Fig. 7). The mammary gland is a highly motile network of branching epithelial tissue that advances and recedes during different stages of post-natal development (i.e. puberty, pregnancy, nursing, etc ${ }^{69}$.). The characteristic directionality of mammary branching is conferred by polarized cell proliferation and phagocytosis mediated by macrophage remodeling, especially in anticipation of nursing ${ }^{70}$. Furthermore, multiple complement and complement-regulatory components are robustly upregulated during periods of apoptosis and phagocytosis in the mammary tissue of multiple species including humans ${ }^{71,72}$, though the functional significance of this regulatory program is unknown. Breast milk itself also suppresses complement activation ${ }^{73}$. Finally, CSMD1 is expressed on the lumenal aspect of mammary ducts and terminal end buds, where much of the pregnancyassociated breast remodeling occurs ${ }^{15,74}$ (Fig. 7b). We observed a reduction in mammary epithelial density, due to reduction in 
secondary or tertiary branch points, whose normal geneses are governed by multiple upregulatory and downregulatory chemotactic signals in concert with physical interaction with phagocytic cells (i.e. macrophages) ${ }^{75}$.

The unmasking of a more severe phenotype in C3/Csmd $1 \mathrm{DKO}$ mice is an unexpected but previously documented signature of complement-mediated disease. For example, double knockout of complement factor $\mathrm{H}(C F H)$ and factor $\mathrm{P}(C F P)$ unexpectedly converts mild C3 glomerulonephritis to lethal C3 glomerulonephritis in mice ${ }^{76}$. Similarly, CFH/C3 DKO unexpectedly unmasks a more severe form of age-related macular degeneration in mice $^{77}$. Multiple explanations for this phenomenon have been set forth, including a dual role of $\mathrm{C} 3$, differences between fluid-phase and local C3 activation, and context-specific C3 gain-of-function. Finally, we cannot exclude the possibility of primary defects outside of the gonads causing pathology in our double knockouts (e.g. altered hemostasis, central defects, etc.). More extensive mutation constructs including conditional knockouts and allelic series may help to distinguish among these scenarios.

Altogether, our human genetic and model organism findings lend support to a role of CSMD1 across multiple tissue types, and unveil a potential role for the complement system in postnatal developmental processes across multiple tissues in the body. When interpreted in context of existing observations from mammalian brain and other model organisms, we predict that macrophage mediated complement activity on self cells is a normal and highly controlled process in many developmental systems in metazoans. Furthermore, a wealth of literature shows that genetic dysregulation of complement and complementregulating factors is a core feature of several heritable diseases beyond infertility; ${ }^{20,58,78-87}$; we therefore consider CSMD1 to also be a reasonable candidate for many of these diseases. Our work highlights the need for deeper investigation into the role of immune system components in reproductive tissues, and the opportunities that such work can have to illuminate and connect common biological processes that produce disease in more complex contexts across the body.

\section{Methods}

Human patient populations. All human genetic studies were reviewed and approved by the Institutional Review Board of Washington University in St. Louis, under protocol numbers \#201107177 and \#201109261 and all participants provided informed consent. Male idiopathic infertility cases were clinically ascertained and pre-screened for chromosome $\mathrm{Y}$ deletions and compared against normospermic controls $^{13,14}$. WHI-SHARe. To create an analogous case-control cohort of female gonadal dysfunction, we turned to the SNP Health Association Resource (SHARe) ${ }^{88}$. We constructed early idiopathic case and control definitions from the dense reproductive phenotype data collected on each subject. A self-reported age of menopause before 40 years was used as the only case inclusion criterion. Case exclusion criteria were oophorectomy prior to age 40, a diagnosis of lupus or rheumatoid disease, and a "yes" answer to the question "Did a doctor ever say that you had cancer, a malignant growth, or tumor?". Smoking history, which is a known factor influencing ovarian reserve, was controlled for during the analysis of genetic data.

UK Biobank. This research has been conducted using the UK Biobank Resource under Application Number '17085'. We generated a table of phenotype data for constructing early idiopathic menopause case and control labels using controlledaccess data from the UK Biobank. Exclusion criteria for the study were: withdrawn consent, poor heterozygosity or missingness as defined by the UK Biobank; $>10$ relatives in the UK Biobank cohort; not used in autosome phasing, apparent sex chromosome aneuploidy; mismatch between genetic and self-reported sex; ever smoker; any self-reported non-Caucasian ancestry; oophorectomy prior to age 40; prior diagnosis of rheumatoid arthritis, lupus, or pelvic cancer; mismatch between self-reported ethnicity or age at menopause among three assessments; SNP array call rate $<98 \%$. In the case of pairs of 2 nd degree relatives or closer, the one individual with the lower SNP-call rate was dropped. The inclusion criteria for POI case status were self-reported age of menopause $<40$ years old, and all remaining individuals in the cohort (after exclusions mentioned above) were used as controls.

Mouse colony breeding. We acquired a constitutive Csmd1 knockout mouse (Mus musculus) on a mixed 129SvEvBrd:C57BL/6 background from the UC Davis
KOMP Repository (Project ID CSD118901) ${ }^{89}$. A $1.086 \mathrm{~kb}$ deletion encompassing Csmd1 exon 1 and part of intron 1 were replaced with a lacZ/neomycin cassette. Deletion of this segment was confirmed with Southern blot and PCR. Due to the extreme size of Csmd1 (1.6 Mb), we also analyzed RNA seq data across all 70 exons in knockout testes and ovaries. In ovaries, knockout read counts relative to wildtype are suppressed across all 70 exons. In testes, knockout read counts relative to wildtype are broadly suppressed across exons 1-57 and upregulated from exons 5870. The amino acid coding portion of these upregulated exons range in size from $45 \mathrm{bp}$ to $180 \mathrm{bp}$. The translational viability of these fragments is unknown. All littermate tissue comparisons in this study (described below) were generated from dam $_{\text {heterozygous }} \times$ sire $_{\text {heterzygous }}$ crossings from this original colony. Next, to eliminate variance in phenotype explained by variance in background genotype (if any), we serially backcrossed the Csmd 1 mutation onto a constant C57BL/6 background for 5 generations. From this F5 backcross generation we performed a dam heterozygous $\times$ sire $_{\text {heterozygous }}$ cross from this to create wildtype and knockout littermates, and performed analogous histology and immunofluorescence experiments as with the original colony (described below; Supplementary Fig. 5e). We performed microsatellite genotyping of these littermates to estimate the C57BL/6 background after backcrossing (Washington University Rheumatic Disease Core). We estimated the F5 proportion of C57BL/6 ancestry of 0.91 (95\% CI [0.89-0.93]). For DKO experiments, we introgressed a $C 3$ mutant line ${ }^{90}$ until we achieved Csmd1/C3 DKO mice. All animal experiments were performed in compliance with the regulations of the Animal Studies Committee at Washington University in St. Louis under protocols \#20120244 and \#20160089.

CNV and SNV discovery. Array data for the Women's Health Initiative SHARe cohort were downloaded from the NCBI Database of Genotypes and Phenotypes (dbGAP accession number phg0000g1.v2). SHARe samples were genotyped on the Affymetrix 6.0 platform. We created a high-quality set of CNV calls for all cohorts using our own internal pipelines. SHARe samples were processed with Affy6CNV (a wrapper that we wrote for the Birdsuite package ${ }^{91}$ ) for data processing and QC We obtained raw SNP array data from the UK Biobank and performed single sample CNV discovery using PennCNV ${ }^{92}$. Individuals with $>200 \mathrm{CNV}$ calls were dropped. CNV calls with PennCNV quality score $>30$ were retained, and adjacent CNVs in the same sample were merged. All qPCR validation of CSMD1 CNV calls were performed on Applied Biosystems TaqMan assay \# Hs0367742_cn (catalog \#4400291). We used Human RNase P for the reference assay (catalog \#4403326). We used standard Applied Biosystems TaqMan protocols in $10 \mu \mathrm{L}$ reaction volumes. We generated 4 to 8 technical replicates for each sample in a 384 well plate. PCR conditions were as follows: $50^{\circ} \mathrm{C}$ for $2 \mathrm{~min}, 95^{\circ} \mathrm{C}$ for $10 \mathrm{~min}$, then 40 cycles of $95^{\circ} \mathrm{C}$ for $15 \mathrm{~s}$ and $60^{\circ} \mathrm{C}$ for $1 \mathrm{~min}$. TaqMan copy number was estimated using the CopyCaller software and compared to PennCNV copy number estimates.

Exome sequencing was performed on a subset of the WHI subjects as part of the Women's Health Initiative Sequencing Project (WHISP); all available WHISP BAM files were downloaded from the NCBI Database of Genotypes and Phenotypes (dbGaP accession phs000200.v10.p3.c1 and phs000200.v10.p3.c2) ${ }^{93}$ Genotypes were recalled, jointly, from 1668 WHI BAM files using Haplotype Caller, recalibrated and cleaned according to GATK best practices using GATK3.2.2.

Association testing. CNVs. Rare CNVs were associated with case-control status using generalized linear models. For the SHARe association analysis, we included the top 10 ancestry eigenvectors (calculated from the full SHARe genotype matrix) and smoking history as covariates. For the UK BioBank association analyses, and we included as covariates BMI, smoking history (or individuals with any history of smoking were excluded, where noted) and the top 10 ancestry eigenvectors calculated from the full UK Biobank SNP genotype matrix.

In addition to idiopathic early menopause (defined above in Human patient populations), we performed association testing for the following 4 traits for UKBB subjects (Unique Data Identifier codes in parentheses): Length of menstrual cycle (3710), Number of live births (2734), Age at last live birth (2764), all-cause female infertility IC10 code (N97)

SNVs. We tested for an association between rare SNVs in CSMD1 and age at menopause in the WHI samples using the Sequence Kernel Association Test $(\text { SKAT })^{94}$, weighting each variant with the Combined Annotation Dependent Depletion (CADD) value ${ }^{95}$. Five ancestry eigenvectors and smoking history were included as covariates; significance was evaluated using bootstrapping with 5000 samples.

Testes dissociation and cell sorting and RNA extraction. We adapted existing methods for dissociation and germ cell purification ${ }^{96}$ which we describe below. Sexually mature ( $40 \pm 1$ days old), male mice were sacrificed, and their testes were decapsulated and homogenized in a $1 \times$ MEM solution (Gibco 11430-030) containing $120 \mathrm{U} / \mathrm{mL}$ Type I Collagenase (Worthington Biochemical LS004194) and $1 \mathrm{mg} / \mathrm{mL}$ DNAse I (Roche 10104159001), and agitated for $15 \mathrm{~min} .1 \times \mathrm{MEM}$ was replaced and added with $50 \mathrm{mg} / \mathrm{mL}$ Trypsin (Worthington Biochemical 54J15037) and $1 \mathrm{mg} / \mathrm{mL}$ DNAse I and agitated for 15 minutes, then mechanically homogenized for $3 \mathrm{~min} .50 \mathrm{mg} / \mathrm{mL}$ Trypsin and $1 \mathrm{mg} / \mathrm{mL}$ DNAse I were added and agitated again for $15 \mathrm{~min}$. We added $0.4 \mathrm{~mL}$ heat inactivated Fetal Bovine Serum 
(Sigma F1051), $5 \mu \mathrm{L}$ Hoescht 33342 (Life Technologies H3570), and $1 \mathrm{mg} / \mathrm{mL}$ DNAse I, and agitated for 15 minutes. Individual cells were dissociated by pipetting sequentially through two $40 \mu \mathrm{m}$ cell strainers (Falcon 352340 ). For each individual mouse, one dissociated testis was used for whole tissue RNA extraction and sequencing, and the other testis was used for germ cell purification, RNA extraction, and sequencing. All dissociation steps were performed at $33^{\circ} \mathrm{C}$. Dissociated testes were sorted on a modified MoFlo cytometer (Beckman Coulter) at the Washington University Siteman Flow Cytometry Core using a krypton-ion laser ${ }^{97}$. Cells that are stained with Hoechst can be clustered in two wavelengths: (i) blue fluorescence, which is informative of DNA content, and (ii) red fluorescence, which is informative about chromatin state and Hoechst efflux from the cell. Our gating strategy is graphically summarized in Supplementary Fig. 3. Based on these parameters, we separated homogenized testes suspensions into four purified populations: (i) spermatogonia, (ii) primary spermatocytes, (iii) secondary spermatocytes, and (iv) spermatids. These separated populations were collected and RNA extraction performed on them. RNA from whole testes was extracted with the RNeasy Plus Mini Kit (Qiagen 74134), and RNA from FACS-purified germ cell populations was extracted with the RNeasy Plus Micro Kit (Qiagen 74034).

RNA-seq. Whole testis, whole ovaries, and purified male germ cell subpopulations were obtained from wildtype and Csmd1 null siblings (summarized in Supplementary Data 3). We extracted polyadenylated mRNAs from each tissue/cell type and converted these into RNA-seq libraries. Three biological replicates of each tissue or cell type were sequenced with a $2 \times 101$ bp paired-end protocol. Reads were mapped to Ensembl Mus musculus reference R72 and transcript expression levels were summarized as reads-per-kb of exon per million-mapped reads (RPKM) using the TopHat2 package ${ }^{98}$. RPKMs were adjusted for batch effects and cryptic covariates using PEER ${ }^{99}$, quantile normalized, and then the $\mathrm{R}$ package poissonSeq was used for differential expression analyses ${ }^{100}$.

Immunostaining and imaging. Testes and ovaries were dissected, fixed in $4 \%$ paraformaldehyde (Electron Microscopy Sciences), and embedded in paraffin. We baked $5 \mu \mathrm{m}$ sections at $60^{\circ} \mathrm{C}$ for $1 \mathrm{~h}$, deparaffinized in Xylenes, and rehydrated into PBS (Corning). Antigen retrieval was done in boiling citrate buffer ( $10 \mathrm{mM}$ sodium citrate, $0.05 \%$ Tween-20, $\mathrm{pH}$ 6.0) for $20 \mathrm{~min}$. Sections were blocked in PBS containing $0.2 \%$ Triton X-100 and 5\% normal donkey serum (Jackson Laboratories) for $1 \mathrm{~h}$ at room temperature and then incubated with primary antibodies diluted in blocking solution over night at $4{ }^{\circ} \mathrm{C}$. After washing with PBS-Tx (PBS containing $0.2 \%$ Triton X-100), they were incubated with fluorescent secondary antibodies in blocking solution for $1 \mathrm{~h}$ at room temperature, washed with PBS-Tx, and treated with $0.2 \%$ Sudan Black in $70 \% \mathrm{EtOH}$ for 10 min, followed by PBS washes. The sections were then counterstained with Hoechst dye 33342 diluted 1:500 in PBS for 5 min, washed once with PBS-Tx for 2 min and then with PBS, and mounted in ProLong Diamond anti-fade mounting medium (Molecular Probes). Imaging was done on an Olympus LSM700 confocal microscope using Zen software, and images were processed using Photoshop CS5 (Adobe). Antibodies used were gt $\alpha$-CSMD1 N20 (Santa Cruz Biotechnology sc-68280 1:100), rb a-mouse vasa homolog (MVH) (Abcam 13840, 1:1,000), donkey $a$-gt CF594 (Biotium \#20116 1:300), and donkey a-rb Alexa488 (Life Technologies \#A-21206 1:300), rb a- $\beta$-gal (Cappel \#55976 1:333), rat F4/80 BM8 (Santa Cruz Biotechnology sc-52664 1:50), donkey a-rat Alexa488 (Life Technologies \#A-21208 1:300), rb $\alpha$-C3 (Abcam 200999, 1:2000), and gt $\alpha$-rb Alexa568 (Life Technologies \#A-11011, 1:300). Testes were decapsulated in 1x PBS and the tubules were gently teased apart and fixed in $4 \%$ paraformaldehyde in $1 \times \mathrm{PBS}$ for $2 \mathrm{~h}$ at $4{ }^{\circ} \mathrm{C}$. After washing with PBS and blocking in PBS containing $0.2 \%$ Triton X-100 and 5\% normal donkey serum for $1.5 \mathrm{~h}$ at room temperature, they were incubated in primary antibodies overnight at $4{ }^{\circ} \mathrm{C}$ in blocking solution and processed as the cross-sectional IF experiments were described above ${ }^{33}$. For immunohistochemistry, $5 \mu \mathrm{m}$ paraffin sections were treated as above, except the secondary antibody was biotin-coupled horse $\alpha$-goat (Vector Laboratories, BA-9500, 1:200), and detection was done using the Vectastain Elite ABC kit (Vector Laboratories, PK-6100) and DAB Peroxidase Substrate kit (Vector Laboratories SK-4100) per the manufacturer's instructions. Sections were counterstained with hematoxylin, mounted in Cytoseal Xyl (Thermo Scientific), and imaged on a Zeiss Axioplan 2 microscope equipped with an Olympus DP71 camera and DP software. For X-gal staining, whole tissues were fixed in 4\% PFA in PBS for $2 \mathrm{~h}$, washed with PBS, and stained in X-gal (GoldBio \#X4281C1) overnight ${ }^{101}$. Tissues were embedded in paraffin, sectioned at $5 \mu \mathrm{m}$, and counterstained with hematoxylin.

Histology. Freshly-dissected gonads were fixed under agitation in Modified Davidson's fixative (Electron Microscopy Sciences 64133-50) for $24 \mathrm{~h}$ and Bouin's fixative (Electron Microscopy Sciences 26367-01) for 24 h. Fixed tissues were embedded in paraffin and sectioned at $5 \mu \mathrm{m}$. Sectioned tissues were stained with hematoxylin and counter-stained with either Periodic acid-Schiff reagent ${ }^{102}$ or eosin. Stained testes from 65 individual mice of known age and genotype (12 wildtype, 53 knockout) were provided to a single mouse pathologist in a blinded fashion. All samples received a score of 0 (no damage), 1 (mild damage), or 2 (severe damage) (see Supplementary Fig. 5a for exemplars). In order to estimate the effect of genotype on score, we fit a linear analysis of variance model:

$$
y_{i j k}=\mu+\alpha_{i}+\beta_{j}+\varepsilon_{i j k}
$$

where $y_{i j k}$ is the damage score for individual $k, \mu$ average damage score across all animals, $\alpha_{i}$ is the effect of genotype $i, \beta_{j}$ is the effect of age $j$, and $\varepsilon_{i j k}$ is the random error associated with the $k$ th observation.

Germ cell quantification. We performed immunofluorescence as described above on a pair of 34 day old male littermates (the same individuals as seen in Supplementary Fig. 5c) using TRA98 antibody (Abcam ab82527 1:200). We generated count data for total cells (filtering based on size and shape), and for TRA98-positive cells (filtering based on green fluorescence) using the ImageJ software package. In order to estimate the effect of genotype on TRA98 cell count, we fit the following model:

$$
\ln \left(y_{i}\right)=\beta_{0}+\beta_{1} X_{1 i}+\beta_{2} X_{2 i}+\varepsilon_{i}
$$

Where $y_{i}$ is the TRA98-positive count in image $i$, and $X_{1}$ is the genotype (Csmdl wildtype versus knockout), and $X_{2}$ is the total cell count. $\varepsilon_{i}$ is the nuisance variable for image $i$.

Gonad size analysis. We sacrificed 229 adult mice (106 males and 123 females), and measured body weights and bilateral gonad weights at necropsy. For males, mean body weight was $37.1 \mathrm{~g}$, mean testes weight was $273 \mathrm{mg}$, and mean age was 201 days. For females, mean body weight was $31.3 \mathrm{~g}$, mean ovary weight was $32 \mathrm{mg}$, and mean age was 234 days. In order to estimate the effect of genotype on gonad weight, we fit the following linear model:

$$
y_{i}=\beta_{0}+\beta_{1} X_{1 i}+\beta_{2} X_{2 i}+\beta_{3} X_{3 i}+\varepsilon_{i}
$$

Where $y_{i}$ is the gonad weight in individual $i$, and $X_{1 i}, X_{2 i}$ and $X_{3 i}$ are the genotype, age, and body weight of individual $i$, respectively. $\varepsilon_{i}$ is the nuisance variable for individual $i$.

Quantifying daily sperm production. Daily sperm production (DSP) was estimated using the Triton-X 100 nuclear solubilization method ${ }^{103}$. Frozen testes were quickly thawed at room temperature and approximately half was used to estimate DSP. Sample weight was registered for full testis and tissue fragment used for the assay. To burst all cells, except for spermatid heads, the tissue was sonicated in DSP buffer $(0.9 \% \mathrm{NaCl}, 0.01 \%$ sodium azide (SAZ), $0.05 \%$ Trition-X-100) using short pulses $(3 \times 10 \mathrm{~s}$ at $30 \%$ amplitude). Sperm heads were then collected by centrifugation $(10 \mathrm{~min}$ at $500 \times \mathrm{g})$ and used in 1:1 dilutions with $0.4 \%$ Trypan Blue for estimation of cell density per homogenate in a hemacytometer. These values were then used to estimate the total number of spermatids per testis [number spermatids in homogenate divided by sample weight (g) and multiplied by the full testis weight (g)]. In mouse spermiogenesis, steps 14-16 take 4.84 days (developing spermatids), therefore, DSP was calculated by dividing the total number of spermatids in one testis per 4.84 days.

Follicle count analysis. We sacrificed 15 sexually mature female mice, of which 10 were wildtype and 5 were knockout genotypes. Bilateral ovaries were fixed, sectioned to $5 \mu \mathrm{m}$, and stained with $\mathrm{H} \& \mathrm{E}$. We identified primordial follicles, primary follicles, secondary follicles, early antral follicles, antral follicles, preovulatory follicles, atretic follicles, and corpora lutea across each section based on histologic appearance ${ }^{104}$. In order to estimate the effect of genotype on gonad weight, we fit the following model:

$$
\ln \left(y_{i}\right)=\beta_{0}+\beta_{1} X_{1 i}+\beta_{2} X_{2 i}+\varepsilon_{i}
$$

Where $y_{i}$ is the number of total oocytes in bilateral ovaries of individual $i$, and $X_{1 i}$ and $X_{2 i}$ are genotype and age, respectively. $\varepsilon_{i}$ is the nuisance variable for individual $i$

Breeding time analysis. We compiled comprehensive husbandry information over a period of greater than 1 year corresponding to 151 litters born representing all possible Csmd1 wildtype, heterozygote, and knockout sire/dam breeding combinations. We calculated the number of days between first sire/dam co-habitation and birth of each litter. Next we subtracted an estimated C57BL/6 gestation time of 19 days ${ }^{105}$ to estimate time to conception. We also calculated parental ages at conception. All density plots depicted in Fig. 5b reflect estimated time to conception for all 151 litters. In order to estimate the effect of maternal genotype on mating success, we controlled for paternal genotype by including wildtype sires only. We then fit the following linear model:

$$
y_{i}=\beta_{0}+\beta_{1} X_{1 i}+\beta_{2} X_{2 i}+\beta_{3} X_{3 i}+\varepsilon_{i}
$$

Where $y_{i}$ is the estimated time to conception for mating pair $i, X_{1 \mathrm{i}}$ is maternal genotype (wildtype, heterozygote, or knockout), $X_{2 i}$ is maternal age at conception, and $X_{3 i}$ is paternal age at conception. $\varepsilon_{i}$ is the nuisance variable.

Litter size analysis. We bred 44 females ( 8 wildtype, 27 heterozygote, and 9 homozygote) with 41 males ( 4 wildtype, 26 heterozygote, and 11 homozygote) over 
a period of 10 months to produce 99 litters, totaling 688 live births. All 9 parental genotype permutations [ $\mathrm{wt}_{\mathrm{dam}} \times \mathrm{wt}_{\text {sire }}, \mathrm{wt}_{\mathrm{dam}} \times$ het $_{\text {sire }} \ldots \mathrm{hom}_{\mathrm{dam}} \times \mathrm{hom}_{\text {sire }}$ ] were represented multiple times (excepting het dam $\times \mathrm{wt}_{\text {sire }}$ ). We counted deaths in during the neonatal period (defined as 1-10 days by convention, although the vast majority of deaths occurred within 24-48 h) and subtracted from the live birth total to obtain the final number of surviving pups (550 total). Next, we stratified each litter by maternal and paternal genotype status (Csmd1 wildtype or heterozygous versus knockout) and fit the following model:

$$
\ln \left(y_{i}\right)=\beta_{0}+\beta_{1} X_{1 i}+\beta_{2} X_{2 i}+\varepsilon_{i}
$$

Where $y_{i}$ is the number of surviving pups in litter $i$, and $X_{1 i}$ and $X_{2 i}$ are the maternal and paternal genotypes, respectively. $\varepsilon_{i}$ is the nuisance variable for litter $i$.

Mammary gland whole-mount analysis. Female littermates were collected at four developmental time points: (i) pre-pubescent ( $<30$ days of age); (ii) adult virgins; (iii) mid-pregnancy (estimated 14 days after copulation); (iv) post-weaning ( 7 days after weaning pups from mother's nursing). Freshly-dissected whole inguinal mammary glands were fixed overnight in Carnoy's solution (60\% ethanol, $30 \%$ chloroform, $10 \%$ glacial acetic acid). Fixed tissues were washed and rehydrated in ethanol and water and stained in Carmine alum histological stain (0.5\% Aluminum potassium sulfate, $0.2 \%$ Carmine) for $48 \mathrm{~h}$. Stained tissues were dehydrated with increasing concentrations of ethanol and stored in xylene to clear lipids for $48 \mathrm{~h}$. Finally, tissues were flattened mechanically and suspended in pure methyl salicylate prior to imaging. Due to the large size of whole mammary tissues, overlapping fields of view were captured and stitched together using the "Photomerge" function in Adobe Photoshop. Gaps in the backdrop of the merged images were filled using the "Content aware fill" function in Adobe Photoshop - if and only if the gaps did not overlap any portion of the tissue proper. All original images are available on the Conrad Lab website. To perform statistical comparison of duct morphology between genotypes, measurements of mammary gland ducts were derived from images using AngioTool64 v0.6a ${ }^{106}$. First, a skeleton representation of the branched duct structure is generated from the input image, which is then used to compute a variety of morphological and spatial parameters for branching characterization. Since this software detects the branches by contrast on a black background, the images of whole mount mammary glands of adult mice were transformed into a compatible input using Image $1.51 \mathrm{n}$.

Hormone measurements. We collected serum from 9 males (4 wildtype versus 5 knockout; mean age $=103$ days) and 16 females in the proestrous stage ( 7 wildtype versus 9 knockout; mean age $=96$ days) via submandibular collection. Each wildtype individual was matched with $\geq 1$ knockout littermate. Female estrous cycle was determined first by external inspection of the vaginal opening, then by vaginal cytology ${ }^{107}$. Vaginal swabs were collected, transferred to a glass slide, and inspected under light microscopy. Proestrus, estrus, metestrus, and diestrus staging was then classified based on the relative counts of leukocytes, nucleated epithelial cells, and cornified epithelial cells. Only confirmed proestrous females were selected for blood draws and hormone measurements. All blood was drawn at approximately the same time of day, clotted for $90 \mathrm{~min}$ at room temperature, and centrifuged at $2000 \times g$ for $15 \mathrm{~min}$. Samples were stored at $-20^{\circ} \mathrm{C}$ prior to hormone measurements. Male samples were quantified for LH/FSH (EMD Millipore) and testosterone (Immuno-Biological Laboratories Inc), and female samples were quantified for LH/FSH and estradiol (CALBIOTECH) by the University of Virginia Ligand Assay and Analysis Core.

C3 deposition assay. Testes obtained post-dissection from Csmd1 knockout and wild-type mice were decapsulated and washed in $1 \times$ PBS before mincing. Minced tissue was subjected to enzymatic dissociation as described above. The crude cell preparation thus obtained was treated with ACK buffer (Life Technologies) for $5 \mathrm{~min}$ at room temperature to lyse erythrocytes present if any in the cell preparation. The isolated cells were incubated in a-C3 (B9) primary antibody (Santa Cruz Biotechnology sc-28294, 1:100) diluted in FACS buffer (1× PBS, 5\%FBS, $0.1 \%$ Sodium azide) along with $10 \% \mathrm{Fc}$ block (to minimize non-specific binding and background fluorescence) for $45 \mathrm{~min}$ at room temperature, followed by gt $\alpha$-mouse Alexa488 (Abcam \#A11029 1:250) incubation of 90 mins at RT in the dark with 3 washes of ice cold FACS buffer after each antibody incubation. Flow cytometry was performed with an Accuri C6 cytometer (BD Biosciences).

Reporting summary. Further information on research design is available in the Nature Research Reporting Summary linked to this article.

\section{Data availability}

Human sample data are from WHI-SHARe (dbGaP Study Accession phs000386.v7.p3), WHISP (dbGaP Study Accession phs000281.v6.p3), UK Biobank (www.ukbiobank.ac.uk), and GEMINI (www.gemini.conradlab.org) and can be obtained upon application. GTEx data were viewed through www.gtexportal.org and ENCODE data were viewed in the UCSC genome browser (genome.ucsc.edu). RNA-seq data generated in this study have been submitted to the Gene Expression Omnibus accession number (GSE136769). We further provide processed RNA-seq RPKM information for all samples as
Supplementary Data. A reporting summary for this Article is available as a Supplementary Information file. The source data underlying Figs. 1-5, 7 and Supplementary Figs. 1, 3, 4, 6, and 9 are provided as a Source Data file. Additional information is available upon request.

\section{Code availability}

Scripts used to perform data analysis are available upon request.

Received: 20 September 2018; Accepted: 11 September 2019; Published online: 11 October 2019

\section{References}

1. O'Flynn O'Brien, K. L., Varghese, A. C. \& Agarwal, A. The genetic causes of male factor infertility: a review. Fertil. Steril. 93, 1-12 (2010).

2. Nelson, L. M. Clinical practice. Primary ovarian insufficiency. N. Engl. J. Med. 360, 606-614 (2009).

3. Hotaling, J. \& Carrell, D. T. Clinical genetic testing for male factor infertility: current applications and future directions. Andrology 2, 339-350 (2014).

4. Stolk, L. et al. Meta-analyses identify 13 loci associated with age at menopause and highlight DNA repair and immune pathways. Nat. Genet. 44, 260-268 (2012).

5. Matzuk, M. M. \& Lamb, D. J. The biology of infertility: research advances and clinical challenges. Nat. Med. 14, 1197-1213 (2008).

6. Sun, Y. C., Sun, X. F., Dyce, P. W., Shen, W. \& Chen, H. The role of germ cell loss during primordial follicle assembly: a review of current advances. Int. J. Biol. Sci. 13, 449-457 (2017).

7. Shaha, C., Tripathi, R. \& Mishra, D. P. Male germ cell apoptosis: regulation and biology. Phil. Trans. R. Soc. B Biol. Sci. 365, 1501-1515 (2010).

8. Hess, R. A. \& Renato de Franca, L. Spermatogenesis and cycle of the seminiferous epithelium. Adv. Exp. Med. Biol. 636, 1-15 (2008).

9. Wallace, W. H. \& Kelsey, T. W. Human ovarian reserve from conception to the menopause. PLoS ONE 5, e8772 (2010).

10. Hsueh, A. J., Billig, H. \& Tsafriri, A. Ovarian follicle atresia: a hormonally controlled apoptotic process. Endocr. Rev. 15, 707-724 (1994).

11. Luborsky, J. L., Meyer, P., Sowers, M. F., Gold, E. B. \& Santoro, N. Premature menopause in a multi-ethnic population study of the menopause transition. Hum. Reprod. 18, 199-206 (2003).

12. Willott, G. M. Frequency of azoospermia. Forensic Sci. Int. 20, 9-10 (1982).

13. Lopes, A. M. et al. Human spermatogenic failure purges deleterious mutation load from the autosomes and both sex chromosomes, including the gene DMRT1. PLoS Genet. 9, e1003349 (2013).

14. Huang, N. et al. A screen for genomic disorders of infertility identifies MAST2 duplications associated with non-obstructive azoospermia in humans. Biol. Reprod. 93, 61 (2015).

15. Kraus, D. M. et al. CSMD1 is a novel multiple domain complement-regulatory protein highly expressed in the central nervous system and epithelial tissues. J. Immunol. 176, 4419-4430 (2006).

16. Liszewski, M. K., Farries, T. C., Lublin, D. M., Rooney, I. A. \& Atkinson, J. P. Control of the complement system. Adv. Immunol. 61, 201-283 (1996).

17. Schafer, D. P. et al. Microglia sculpt postnatal neural circuits in an activity and complement-dependent manner. Neuron 74, 691-705 (2012).

18. Schizophrenia Psychiatric Genome-Wide Association Study C. Genome-wide association study identifies five new schizophrenia loci. Nat. Genet. 43, 969-976 (2011).

19. Schizophrenia Working Group of the Psychiatric Genomics C. Biological insights from 108 schizophrenia-associated genetic loci. Nature 511, 421-427 (2014).

20. Sekar, A. et al. Schizophrenia risk from complex variation of complement component 4. Nature 530, 177-183 (2016).

21. Zhao, H. et al. A genome-wide association study reveals that variants within the HLA region are associated with risk for nonobstructive azoospermia. Am. J. Hum. Genet. 90, 900-906 (2012).

22. $\mathrm{Ni}, \mathrm{B}$. et al. Low-frequency germline variants across $6 \mathrm{p} 22.2-6 \mathrm{p} 21.33$ are associated with non-obstructive azoospermia in Han Chinese men. Hum. Mol. Genet. 24, 5628-5636 (2015).

23. Escudero-Esparza, A., Kalchishkova, N., Kurbasic, E., Jiang, W. G. \& Blom, A. $\mathrm{M}$. The novel complement inhibitor human CUB and Sushi multiple domains 1 (CSMD1) protein promotes factor I-mediated degradation of C4b and C3b and inhibits the membrane attack complex assembly. FASEB J. 27, 5083-5093 (2013).

24. Chen, C. T. et al. Replication of loci influencing ages at menarche and menopause in Hispanic women: the Women's Health Initiative SHARe Study. Hum. Mol. Genet. 21, 1419-1432 (2012).

25. Sudlow, C. et al. UK biobank: an open access resource for identifying the causes of a wide range of complex diseases of middle and old age. PLoS Med. 12, e1001779 (2015). 
26. Bochukova, E. G. et al. Large, rare chromosomal deletions associated with severe early-onset obesity. Nature 463, 666-670 (2010).

27. Perry, J. R. et al. Parent-of-origin-specific allelic associations among 106 genomic loci for age at menarche. Nature 514, 92-97 (2014).

28. Day, F. R. et al. Genomic analyses identify hundreds of variants associated with age at menarche and support a role for puberty timing in cancer risk. Nat. Genet. 49, 834-841 (2017).

29. Day, F. R. et al. Large-scale genomic analyses link reproductive aging to hypothalamic signaling, breast cancer susceptibility and BRCA1-mediated DNA repair. Nat. Genet. 47, 1294-1303 (2015).

30. Soumillon, M. et al. Cellular source and mechanisms of high transcriptome complexity in the mammalian testis. Cell Rep. 3, 2179-2190 (2013).

31. Steen, V. M. et al. Neuropsychological deficits in mice depleted of the schizophrenia susceptibility gene CSMD1. PLoS ONE 8, e79501 (2013).

32. Li, R. \& Albertini, D. F. The road to maturation: somatic cell interaction and self-organization of the mammalian oocyte. Nat. Rev. Mol. Cell Biol. 14, 141-152 (2013).

33. DeFalco, T. et al. Macrophages contribute to the spermatogonial niche in the adult testis. Cell Rep. 12, 1107-1119 (2015).

34. Lie, P. P., Mruk, D. D., Lee, W. M. \& Cheng, C. Y. Cytoskeletal dynamics and spermatogenesis. Phil. Trans. R. Soc. B Biol. Sci. 365, 1581-1592 (2010).

35. Smemo, S. et al. Obesity-associated variants within FTO form long-range functional connections with IRX3. Nature 507, 371-375 (2014).

36. Distler, M. G., Opal, M. D., Dulawa, S. C. \& Palmer, A. A. Assessment of behaviors modeling aspects of schizophrenia in Csmd1 mutant mice. PLoS ONE 7, e51235 (2012).

37. Lipkin, S. M. et al. Meiotic arrest and aneuploidy in MLH3-deficient mice. Nat. Genet. 31, 385-390 (2002).

38. Yatsenko, A. N. et al. X-linked TEX11 mutations, meiotic arrest, and azoospermia in infertile men. N. Engl. J. Med. 372, 2097-2107 (2015).

39. Koebele, S. V. \& Bimonte-Nelson, H. A. Modeling menopause: the utility of rodents in translational behavioral endocrinology research. Maturitas 87, 5-17 (2016).

40. Foldi, C. J., Eyles, D. W., McGrath, J. J. \& Burne, T. H. The effects of breeding protocol in C57BL/6J mice on adult offspring behaviour. PLoS ONE 6, e18152 (2011).

41. Wallace, W. H. \& Kelsey, T. W. Ovarian reserve and reproductive age may be determined from measurement of ovarian volume by transvaginal sonography. Hum. Reprod. 19, 1612-1617 (2004).

42. Hansen, K. R. et al. A new model of reproductive aging: the decline in ovarian non-growing follicle number from birth to menopause. Hum. Reprod. 23, 699-708 (2008).

43. Briley, S. M. et al. Reproductive age-associated fibrosis in the stroma of the mammalian ovary. Reproduction 152, 245-260 (2016).

44. Wu, R., Van der Hoek, K. H., Ryan, N. K., Norman, R. J. \& Robker, R. L. Macrophage contributions to ovarian function. Hum. Reprod. Update 10, 119-133 (2004).

45. Perricone, R. et al. Functionally active complement is present in human ovarian follicular fluid and can be activated by seminal plasma. Clin. Exp. Immunol. 89, 154-157 (1992).

46. Chow, W. N. et al. Complement 3 deficiency impairs early pregnancy in mice. Mol. Reprod. Dev. 76, 647-655 (2009).

47. de Vries, L. et al. Exome sequencing reveals SYCE1 mutation associated with autosomal recessive primary ovarian insufficiency. J. Clin. Endocrinol. Metab. 99, E2129-E2132 (2014).

48. Smirin-Yosef, P. et al. A biallelic mutation in the homologous recombination repair gene SPIDR is associated with human gonadal dysgenesis. J. Clin. Endocrinol. Metab. 102, 681-688 (2017).

49. Rigau, M., Juan, D., Valencia, A. \& Rico, D. Intronic CNVs and gene expression variation in human populations. PLoS Genet. 15, e1007902 (2019).

50. Consortium, G. T. et al. Genetic effects on gene expression across human tissues. Nature 550, 204-213 (2017).

51. Consortium, E. P. An integrated encyclopedia of DNA elements in the human genome. Nature 489, 57-74 (2012).

52. Petrovski, S., Wang, Q., Heinzen, E. L., Allen, A. S. \& Goldstein, D. B. Genic intolerance to functional variation and the interpretation of personal genomes. PLoS Genet. 9, e1003709 (2013).

53. Karczewski, K. J. et al. Variation across 141,456 human exomes and genomes reveals the spectrum of loss-of-function intolerance across human proteincoding genes. Preprint at bioRxiv https://doi.org/10.1101/531210 (2019).

54. Goldmann, J. M. et al. Parent-of-origin-specific signatures of de novo mutations. Nat. Genet. 48, 935-939 (2016).

55. Jonsson, H. et al. Parental influence on human germline de novo mutations in 1,548 trios from Iceland. Nature 549, 519-522 (2017).

56. El-Brolosy, M. A. \& Stainier, D. Y. R. Genetic compensation: a phenomenon in search of mechanisms. PLoS Genet. 13, e1006780 (2017).

57. Lau, W. L. \& Scholnick, S. B. Identification of two new members of the CSMD gene family. Genomics 82, 412-415 (2003).
58. Hong, S. et al. Complement and microglia mediate early synapse loss in Alzheimer mouse models. Science 352, 712-716 (2016).

59. Mizuno, M., Harris, C. L. \& Morgan, B. P. Spermatogenic cells distal to the blood-testis barrier in rats lack C3 convertase regulators and may be at risk of complement-mediated injury. J. Reprod. Immunol. 69, 23-34 (2006).

60. Frungieri, M. B. et al. Number, distribution pattern, and identification of macrophages in the testes of infertile men. Fertil. Steril. 78, 298-306 (2002).

61. Holstein, A. F. Spermatophagy in the seminiferous tubules and excurrent ducts of the testis in Rhesus monkey and in man. Andrologia 10, 331-352 (1978).

62. Allan, D. J., Harmon, B. V. \& Roberts, S. A. Spermatogonial apoptosis has three morphologically recognizable phases and shows no circadian rhythm during normal spermatogenesis in the rat. Cell Prolif. 25, 241-250 (1992).

63. Stevens, B. et al. The classical complement cascade mediates CNS synapse elimination. Cell 131, 1164-1178 (2007).

64. Pompon, J. \& Levashina, E. A. A new role of the mosquito complement-like cascade in male fertility in Anopheles gambiae. PLoS Biol. 13, e1002255 (2015).

65. Morales, C. et al. Different patterns of structural luteolysis in the human corpus luteum of menstruation. Hum. Reprod. 15, 2119-2128 (2000).

66. Cohen, P. E., Zhu, L. \& Pollard, J. W. Absence of colony stimulating factor-1 in osteopetrotic (csfmop/csfmop) mice disrupts estrous cycles and ovulation. Biol. Reprod. 56, 110-118 (1997).

67. Gaytan, F., Morales, C., Bellido, C., Aguilar, E. \& Sanchez-Criado, J. E. Ovarian follicle macrophages: is follicular atresia in the immature rat a macrophage-mediated event? Biol. Reprod. 58, 52-59 (1998).

68. Kato, S., Shiratsuchi, A., Nagaosa, K. \& Nakanishi, Y. Phosphatidylserine- and integrin-mediated phagocytosis of apoptotic luteal cells by macrophages of the rat. Dev. Growth Differ. 47, 153-161 (2005).

69. Sternlicht, M. D. Key stages in mammary gland development: the cues that regulate ductal branching morphogenesis. Breast Cancer Res. 8, 201 (2006).

70. Pollard, J. W. Trophic macrophages in development and disease. Nat. Rev. Immunol. 9, 259-270 (2009).

71. Clarkson, R. W., Wayland, M. T., Lee, J., Freeman, T. \& Watson, C. J. Gene expression profiling of mammary gland development reveals putative roles for death receptors and immune mediators in post-lactational regression. Breast Cancer Res. 6, R92-R109 (2004)

72. Laufer, J. et al. Local complement genes expression in the mammary gland: effect of gestation and inflammation. Pediatr. Res. 46, 608-612 (1999).

73. Ogundele, M. O. Anti-complement activities of human breast-milk. Inflamm Res. 48, 437-445 (1999).

74. Kamal, M. et al. Loss of CSMD1 expression is associated with high tumour grade and poor survival in invasive ductal breast carcinoma. Breast Cancer Res. Treat. 121, 555-563 (2010).

75. Ingman, W. V., Wyckoff, J., Gouon-Evans, V., Condeelis, J. \& Pollard, J. W Macrophages promote collagen fibrillogenesis around terminal end buds of the developing mammary gland. Dev. Dyn. 235, 3222-3229 (2006).

76. Lesher, A. M. et al. Combination of factor $\mathrm{H}$ mutation and properdin deficiency causes severe C3 glomerulonephritis. J. Am. Soc. Nephrol. 24, 53-65 (2013).

77. Hoh Kam, J., Lenassi, E., Malik, T. H., Pickering, M. C. \& Jeffery, G. Complement component $\mathrm{C} 3$ plays a critical role in protecting the aging retina in a murine model of age-related macular degeneration. Am. J. Pathol. 183, 480-492 (2013).

78. Yates, J. R. et al. Complement C3 variant and the risk of age-related macular degeneration. N. Engl. J. Med. 357, 553-561 (2007).

79. Haines, J. L. et al. Complement factor $\mathrm{H}$ variant increases the risk of agerelated macular degeneration. Science 308, 419-421 (2005).

80. Warwicker, P. et al. Genetic studies into inherited and sporadic hemolytic uremic syndrome. Kidney Int. 53, 836-844 (1998).

81. Richards, A. et al. Mutations in human complement regulator, membrane cofactor protein (CD46), predispose to development of familial hemolytic uremic syndrome. Proc. Natl Acad. Sci. USA 100, 12966-12971 (2003).

82. Kavanagh, D. et al. Characterization of mutations in complement factor I (CFI) associated with hemolytic uremic syndrome. Mol. Immunol. 45, 95-105 (2008).

83. Fremeaux-Bacchi, V. et al. Mutations in complement C3 predispose to development of atypical hemolytic uremic syndrome. Blood 112, 4948-4952 (2008).

84. Goicoechea de Jorge, E. et al. Gain-of-function mutations in complement factor B are associated with atypical hemolytic uremic syndrome. Proc. Natl Acad. Sci. USA 104, 240-245 (2007).

85. Stoppa-Lyonnet, D. et al. Altered C1 inhibitor genes in type I hereditary angioedema. N. Engl. J. Med. 317, 1-6 (1987).

86. Gale, D. P. et al. Identification of a mutation in complement factor H-related protein 5 in patients of Cypriot origin with glomerulonephritis. Lancet 376, 794-801 (2010) 
87. Ozen, A. et al. CD55 deficiency, early-onset protein-losing enteropathy, and thrombosis. N. Engl. J. Med. 377, 52-61 (2017).

88. Hays, J. et al. The Women's Health Initiative recruitment methods and results. Ann. Epidemiol. 13, S18-S77 (2003).

89. Friddle, C. J. et al. High-throughput mouse knockouts provide a functional analysis of the genome. Cold Spring Harb. Symp. Quant. Biol. 68, 311-315 (2003).

90. Circolo, A. et al. Genetic disruption of the murine complement C3 promoter region generates deficient mice with extrahepatic expression of C3 mRNA. Immunopharmacology 42, 135-149 (1999).

91. Korn, J. M. et al. Integrated genotype calling and association analysis of SNPs, common copy number polymorphisms and rare CNVs. Nat. Genet. 40, 1253-1260 (2008).

92. Wang, K. et al. PennCNV: an integrated hidden Markov model designed for high-resolution copy number variation detection in whole-genome SNP genotyping data. Genome Res. 17, 1665-1674 (2007).

93. Tryka, K. A. et al. NCBI's database of genotypes and phenotypes: dbGaP. Nucleic Acids Res. 42, D975-D979 (2014).

94. Lee, S. et al. Optimal unified approach for rare-variant association testing with application to small-sample case-control whole-exome sequencing studies. Am. J. Hum. Genet. 91, 224-237 (2012).

95. Kircher, M. et al. A general framework for estimating the relative pathogenicity of human genetic variants. Nat. Genet. 46, 310-315 (2014).

96. Bastos, H. et al. Flow cytometric characterization of viable meiotic and postmeiotic cells by Hoechst 33342 in mouse spermatogenesis. Cytometry A 65, 40-49 (2005)

97. Lima, A. C. et al. Multispecies purification of testicular germ cells. Biol. Reprod. 95, 85 (2016).

98. Kim, D. et al. TopHat2: accurate alignment of transcriptomes in the presence of insertions, deletions and gene fusions. Genome Biol. 14, R36 (2013).

99. Stegle, O., Parts, L., Piipari, M., Winn, J. \& Durbin, R. Using probabilistic estimation of expression residuals (PEER) to obtain increased power and interpretability of gene expression analyses. Nat. Protoc. 7, 500-507 (2012).

100. Li, J., Witten, D. M., Johnstone, I. M. \& Tibshirani, R. Normalization, testing, and false discovery rate estimation for RNA-sequencing data. Biostatistics 13, 523-538 (2012).

101. Li, S., Zhou, W., Doglio, L. \& Goldberg, E. Transgenic mice demonstrate a testis-specific promoter for lactate dehydrogenase, LDHC. J. Biol. Chem. 273, 31191-31194 (1998).

102. Dunleavy, J. E. M. et al. Katanin-like 2 (KATNAL2) functions in multiple aspects of haploid male germ cell development in the mouse. PLoS Genet. 13, e1007078 (2017)

103. Cotton, L. et al. FGFR-1 [corrected] signaling is involved in spermiogenesis and sperm capacitation. J. Cell Sci. 119, 75-84 (2006).

104. Myers, M., Britt, K. L., Wreford, N. G., Ebling, F. J. \& Kerr, J. B. Methods for quantifying follicular numbers within the mouse ovary. Reproduction 127, 569-580 (2004).

105. Murray, S. A. et al. Mouse gestation length is genetically determined. PLoS ONE 5, e12418 (2010).

106. Zudaire, E., Gambardella, L., Kurcz, C. \& Vermeren, S. A computational tool for quantitative analysis of vascular networks. PLoS ONE 6, e27385 (2011).

107. Byers, S. L., Wiles, M. V., Dunn, S. L. \& Taft, R. A. Mouse estrous cycle identification tool and images. PLoS ONE 7, e35538 (2012).

\section{Acknowledgements}

We thank all patients and study participants. D.F.C is supported by the National Institutes of Health (R01HD078641 and R01MH101810). A.S.L. was supported by a Distinguished Scholar Award from Washington University School of Medicine. A.C.L. was supported by a fellowship from the Lalor Foundation. We thank Heather Lawson for training and consultation on animal husbandry. We thank Brianne Tabers for technical assistance with animal husbandry. We thank Anne O'Connor and Jo Merriner for performing histology on testis samples, and helpful discussions. We thank Kelle Moley and members of her laboratory (Praba Esakky, Michaela Reid, and Jessica Saben) for consultations on tissue preparations. We thank Tim Schedl, Matt Baum, and Nicole
Rockweiler for helpful discussions. We thank Nicholas Ho for technical assistance with image quantification. We thank Seungeun Lee for discussion of SKAT results. We thank Bill Eades and Chris Holley at the Washington University Siteman Flow Cytometry Core (NCI P30 CA91842) FACS services. We thank the Washington University Rheumatic Disease Core (NIHP30AR48335) for providing backcrossed mouse genotypes. We thank the University of Virginia Ligand Assay and Analysis Core (U54 DH28934) for hormone measurements. The WHI program is funded by the National Heart, Lung, and Blood Institute, National Institutes of Health, U.S. Department of Health and Human Services through contracts HHSN268201100046C, HHSN268201100001C, HHSN268201100002C, HHSN268201100003C, HHSN268201100004C, and HHSN271201100004C. This paper was not prepared in collaboration with investigators of the WHI, has not been reviewed and/or approved by the Women's Health Initiative (WHI), and does not necessarily reflect the opinions of the WHI investigators or the NHLBI. Funding for WHI SHARe genotyping was provided by NHLBI Contract N02HL-64278. The Women's Health Initiative Sequencing Project (WHISP) was funded by Grant Number RC2 HL102924. This study used data from the NHLBI Grand Opportunity Exome Sequencing Project (GOESP). Funding for GO-ESP was provided by NHLBI grants RC2 HL103010 (HeartGO), RC2 HL102923 (LungGO), and RC2 HL102924 (WHISP). The exome sequencing was performed through NHLBI grants RC2 HL102925 (BroadGO) and RC2 HL102926 (SeattleGO).

\section{Author contributions}

D.F.C. devised the study. A.S.L. and D.F.C. led the experimental design. A.S.L. and D.F.C led the data analysis. A.S.L., A.C.L., M.L., R.M., N.H., K.A.V., R.E.W., J.P.A., M.K.O., and D.F.C. performed data analyses. K.I.A. and D.T.C. contributed to the azoospermia replication study and experimental validation of $\mathrm{CNV}$ calls. A.S.L., J.R., A.U., A.C.L., X.W., and R.A.H. performed experiments. A.S.L. and D.F.C. wrote the paper. All authors read and approved the paper.

\section{Competing interests}

The authors declare no competing interests.

\section{Additional information}

Supplementary information is available for this paper at https://doi.org/10.1038/s41467019-12522-w.

Correspondence and requests for materials should be addressed to D.F.C.

Peer review information Nature Communications thanks Joris Veltman and the other, anonymous, reviewer(s) for their contribution to the peer review of this work. Peer reviewer reports are available.

Reprints and permission information is available at http://www.nature.com/reprints

Publisher's note Springer Nature remains neutral with regard to jurisdictional claims in published maps and institutional affiliations.

Open Access This article is licensed under a Creative Commons Attribution 4.0 International License, which permits use, sharing, adaptation, distribution and reproduction in any medium or format, as long as you give appropriate credit to the original author(s) and the source, provide a link to the Creative Commons license, and indicate if changes were made. The images or other third party material in this article are included in the article's Creative Commons license, unless indicated otherwise in a credit line to the material. If material is not included in the article's Creative Commons license and your intended use is not permitted by statutory regulation or exceeds the permitted use, you will need to obtain permission directly from the copyright holder. To view a copy of this license, visit http://creativecommons.org/ licenses/by/4.0/

(c) The Author(s) 2019 